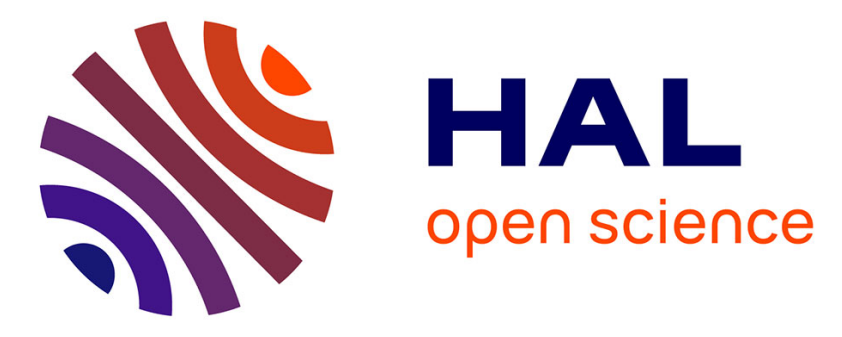

\title{
Verification and Validation of CFD for Surface Combatant 5415 for Straight Ahead and 20 Degree Static Drift Conditions
}

S Bhushan, H Yoon, F Stern, Emmanuel Guilmineau, Michel Visonneau, S Toxopeus, C Simonsen, S Aram, S-E Kim, G Grigoropoulos

\section{To cite this version:}

S Bhushan, H Yoon, F Stern, Emmanuel Guilmineau, Michel Visonneau, et al.. Verification and Validation of CFD for Surface Combatant 5415 for Straight Ahead and 20 Degree Static Drift Conditions. World Maritime Technology Conference, WMTC15, Nov 2015, Rhode Island, United States. hal-02572085

\section{HAL Id: hal-02572085 \\ https://hal.science/hal-02572085}

Submitted on 13 May 2020

HAL is a multi-disciplinary open access archive for the deposit and dissemination of scientific research documents, whether they are published or not. The documents may come from teaching and research institutions in France or abroad, or from public or private research centers.
L'archive ouverte pluridisciplinaire HAL, est destinée au dépôt et à la diffusion de documents scientifiques de niveau recherche, publiés ou non, émanant des établissements d'enseignement et de recherche français ou étrangers, des laboratoires publics ou privés. 


\title{
Verification and Validation of CFD for Surface Combatant 5415 for Straight Ahead and 20 Degree Static Drift Conditions
}

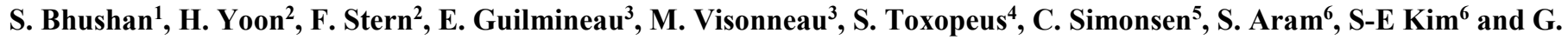 \\ Grigoropoulos $^{7}$
}

${ }^{1}$ Mississippi State University, US; ${ }^{2}$ University of Iowa, US; ${ }^{3} \mathrm{CNRS} / \mathrm{Centrale}$ Nantes, France; ${ }^{4}$ Maritime Research Institute, Netherlands; ${ }^{5}$ FORCE Technology, Denmark; ${ }^{6}$ Naval Surface Warfare Center Carderock Division, US; ${ }^{7}$ National Technical Univ. of Athens, Greece

\begin{abstract}
Collaboration is described on verification and validation of CFD for surface combatant model 5415 for static drift $\beta=0$ and 20 degrees using recent experimental data for: forces and moment, wave elevations, and tomographic PIV planar and volume measurements of velocity, vorticity and turbulent kinetic energy (TKE), including analysis of vortex onset of separation, progression, instability and TKE budget. Results were obtained from five different solvers, which used different numerical and discretization schemes, free-surface and turbulence models and adapted grids ranging from $2.5 \mathrm{M}$ to $102 \mathrm{M}$ for $\beta=0^{\circ}$ and $4.6 \mathrm{M}$ to $250 \mathrm{M}$ for $\beta=20^{\circ}$. For $\beta=0^{\circ}$, resistance and wave-elevation predictions compare within $2 \%$ and $3.5 \%$ of the data, respectively. Solvers agreed with the data for the onset of the primary vortices, but showed large variation in their progression and decay. URANS turbulence models predicted premature decay of vortices, whereas DES predicted too strong vortical structures and low resolved turbulence. The primary vortices exhibited open-type separation. For $\beta=20^{\circ}$, forces and moment compared within $3.7 \%$ of the data, and wave-elevation within $8.5 \%$ of data. Simulations agreed with the data for the onset of primary vortices, but showed large variations for their progression and decay, and for the leeward sonar dome separation bubble and breaking waves. DES performed better than URANS for the prediction of vortex strength and TKE. The vortices show many open-, closed- or open-closed type separations. The primary vortices show helical mode instability, and the instability frequency for the sonar dome tip vortex at $x / L_{P P}=0.4$ compared within $11.3 \% D$ of the data. TKE budget revealed that the production occurs at the vortex inception and is transported by pressure or turbulent fluctuations. The finite-difference solver provided better vortex decay predictions than finite-volume solvers for $\beta=0^{\circ}$. Level-set and VoF provided similar wave elevation predictions except for breaking waves. The study indicates the need for more accurate turbulence closures. Future research should focus on investigation of: improved RANS models such as Reynolds stress transport model; and improved hybrid RANS/LES models to address the turbulence trigger and modeled stress depletion issues of DES.
\end{abstract}

KEY WORDS: Surface Combatant, Verification and Validation, Turbulence Models.

\section{INTRODUCTION}

Prediction of 3D separation including onset and progression of vortical structures is a grand challenge for ship hydrodynamics due to relatively bluff hull forms with attendant thick boundary layer and wake; appendages such as bow bulb/sonar dome, shafts/struts etc.; free-surface/Froude number $(F r)$ effects; and high Reynolds numbers $(R e)$.

The calm water straight-ahead condition has been a central topic of CFD Workshops: most recently Gothenburg 2010 (Larsson et al., 2014; Bhushan et al., 2014). The study concluded that for large block coefficient $C_{B}=0.81 \mathrm{KVLCC} 2$ tanker hull, unsteady Reynolds averaged Navier Stokes (URANS) under predicted the main and secondary vortex sizes and over predicted turbulent kinetic energy (TKE). Detached eddy simulation (DES) (Spalart, 2009) on large 305M grid predicted unsteady vortical structures with $>95 \%$ resolved turbulence levels. They over predicted both axial velocity, and vortical and turbulent structures. Overall, best DES predictions were found to be comparable to best URANS predictions. The poor predictions for the turbulence variables were partially attributed to dated experimental data. Predictions for smaller $C_{B}=0.51$ surface combatant 5415 hull showed a complexity of the onset and progression of sonar dome (SDV) and fore-body keel (FBKV) vortices and inherent free-surface effects $(F r=0.28)$. Prior to the workshop, only SDV had been identified based on sparse experimental data and RANS. A second vortex was measured at $x / L_{P P}=0.6$ (Olivieri et al., 2001), but not observed in the RANS simulations. DES and URANS predictions agreed on the onset of the vortices; however, showed differences on their progression/ interaction. Overall conclusions were that anisotropic URANS models are better than the isotropic models for the prediction of turbulent and vortical structures, but both were too dissipative compared to the experimental data even for grids with more than $10 \mathrm{M}$ cells. In addition URANS results did not show improvements even with $4^{\text {th }}$ order convection schemes. Hybrid RANS/LES models were promising in providing the details of the flow topology, but show modeled stress depletion and grid induced separation in the boundary layer issues for bluff bodies and under resolved turbulence issues for slender bodies. However, a concrete conclusion could not be drawn as the grids were not sufficiently fine to separate the errors due to grid 
resolution and turbulence modeling. Further, a detailed validation of the CFD results was not possible as the available experimental data was dated, sparse, and/or lacking turbulence measurements.

The calm water static and dynamic maneuvering conditions have been the topic of Maneuvering Simulation Workshops, using URANS methods: most recently was SIMMAN 2008 (Stern et al., 2011). The study reported relatively small comparison errors $E \leq$ $5 \% D$, where $D$ is experimental data, for surge $X$ and sway $Y$ forces and yaw moment $\mathrm{N}$ for small drift angle $\beta \leq 10$ degree. But large $E \sim 10 \% D$ were obtained for larger $\beta=20$ degree. For dynamic maneuvers, the trends were similar for larger sway and yaw rates. Local flow analysis used towing-tank PIV measurements for pure sway and yaw at moderate rates showed good agreement for the mean flow, but the vortices were too dissipated. The overall conclusion was that large $\mathrm{E}$ in forces and moments could be due to insufficiency of turbulence models and/or grid resolution.

A related collaborative verification and validation study focused on the flow around the bare hull DARPA SUBOFF in rotational motion (Toxopeus et al., 2012). Primary aim was to predict the loads on the hull as a function of drift angle and rotation rate, to predict the vortices shed from the smooth hull and to crosscompare the different computations to better understand the influence of grid topology, grid resolution and computational settings. The study reported that simulations errors are dominated by modeling errors due to turbulence models or settings. The study showed that with sufficiently fine grids and the selection of advanced turbulence models, the flow around the hull could be predicted well. Results obtained with the standard SST model (Menter, 1994) were found to be inferior to results with corrections to this model or with more advanced turbulence models (e.g. Reynolds stress transport, RSM, models).

CFDShip-Iowa studies were also performed to investigate vortical and turbulent structures for $\operatorname{KVLCC} 2(F r=0)$ at $\beta=0,12$ and 30 degrees using DES and URANS (Xing et al., 2012). The primary focus was an exploratory study for $\beta=30$ degree building on the study of unsteady wave-induced separation for a NACA24 surface-piercing foil (Xing et al., 2007). The study identified one shear layer, one Karman-like vortex shedding, and three helical mode instabilities in the flows. The study reported that the vortices exhibit helical mode instability, show characteristics for vortex breakdown and have a Strouhal number similar to those for delta wings leading edge vortex.

A precursor of the study is Bhushan et al. (2011). The study performed scalability studies for CFDShip-Iowa for large grids (till that date) using both URANS and DES for 5415. The simulations included half domain barehull 5415 at straight-ahead condition for up to $300 \mathrm{M}$ grid, and full domain 5415 with bilge keels at static drift $\beta=20$ degrees for up $250 \mathrm{M}$ grid. The straightahead results were presented in the G2010 workshop. DES for $\beta=$ 20 degrees were motivated by the large drift angle errors at SIMMAN, and focused on simulation with advanced turbulence model, such as DES, and finer grids. The study provided a detailed analysis of the vortical structures, instabilities, and turbulent structures. The fine grid simulations with high levels of resolved turbulence, and less dependence on turbulence modeling, displayed reduced errors for forces and moment, i.e., errors reduced to less than $5 \% \mathrm{D}$ compared to $10 \% \mathrm{D}$ reported at SIMMAN 2008. The unsteady vortex separation and helical model instabilities were similar to those observed for KVLCC2 at $\beta=30$ degrees. However, they showed differences for the onset of the instability, which is discussed in details herein.

Also of relevance is the Gorski et al. $(2002,2004)$ RANS study of the effects of bow dome modifications and to provide information on the inflow to the propulsor both for bare hull and with shafts and struts for surface combatant hull forms using the UNCLE code with up to $16 \mathrm{M}$ cells. The Gorski et al. (2002) concluded that the modifications to the bow dome for Gov't-2 are an improvement over the original geometry. Comparisons with experimental data for the final design were performed for validation purposes demonstrating the accuracy of the RANS predictions. Gorski et al. (2004) performed computations for an aircraft carrier and 5415, and demonstrate that the propeller shafts and struts have a significant effect on the propeller inflow as compared to bare hull configurations. This is demonstrated at both model and full-scale Reynolds numbers with the model scale computations comparing well with experimental data. Without the propeller shaft the dominant effect is the forward bilge and bow dome vortex, particularly at model scale. For calculations at full scale, the flow entering the propellers is very nearly inviscid without the shafts present. The shaft wake is shown to have a significant impact on the flow entering the propeller and shaft rotation further impacts this inflow. A comparison of the propeller inflow for Gov't2 and 5415 indicates significantly larger vortex boundary interaction for former due to its larger bow dome.

Present research is a part of an international collaboration among seven institutions from five different countries focusing on the assessment of the prediction capabilities of state-of-theart Ship hydrodynamics solvers for onset and progression of 3D vortex separation for sea vehicles. The research focused on experimental and computational studies for four benchmark test cases: combatant 5415 for 0 and $20 \mathrm{deg}$. drift angle, for which the experimental data were presented in Yoon et al. (2014) and CFD results are presented herein; high-speed multihull Delft catamaran (DC) for small and large drift angle and $F r$ (Broglia et al., 2015); and tanker KVLCC2 wind tunnel and shallow water for $30 \mathrm{deg}$. drift angle (Abel-Maksoud et al., 2015). Preliminary blind simulations guided the experimental setup for 5415 (Bhushan et al., 2011) and KVLCC2 (Xing et al., 2012) to identify regions of interest and important validation variables. The study was motivated by the lack of prediction capability for large drift angles and yaw rates and lack of physical understanding and prediction capability for surface combatant with sonar domes, as identified in previous ship hydrodynamics CFD workshops (Stern et al., 2011; Larsson et al., 2014) and Task Group AVT-161 Sea Facet activities.

The objective of this paper is to perform and document the verification and validation of CFD numerical methods and models for 5415 at $\beta=0$ and 20 degrees drift conditions, including understanding the physics of vortex onset, separation 
and progression, and assessment of simulation grid requirements and turbulence and free-surface modelling capabilities. The validation study includes assessment of both integral and local flow field details using concurrently procured towing-tank tomographic PIV experimental data (Yoon et al., 2014). The validation data included: forces and moment; wave elevation and profiles; vortical structures, and their axial and cross-plane flow distribution; mean flow, vorticity and turbulence at planar and volume cross-sections along the hull; and dominant flow instabilities. CFD simulations provided vortex onset, separation and instability, and turbulence budget analysis.

\section{EXPERIMENTAL DATA AND CFD COMPUTATIONS}

\section{Experimental Conditions, Validation Variables and Uncertainties}

The hull form used in the tests was the DTMB 5512, a 1:46.6 scale, $3.048 \mathrm{~m}$ long, fiber-reinforced Plexiglas hull, manufactured by the Naval Surface Warfare Center. DTMB 5512 is a geosim of DTMB 5415. The full-scale hull form is a preliminary design for a surface combatant for the US Navy. Characteristic features of the DTMB 5415 hull include a transom stern and a sonar dome bow. The model was unappended except for bilge-keels. The model particulars, the coordinate system and measurement locations along the hull are shown in Fig. 1.

The experiments were conducted in the towing-tank facility at IIHR, University of Iowa. The towing-tank is $100 \mathrm{~m}$ long, $3.048 \mathrm{~m}$ wide and $3.048 \mathrm{~m}$ deep, and is equipped with a wave-maker, a drive carriage, a planar motion mechanism (PMM) carriage, a model motion tracking system, a wall-mounted wave-damping system and a wave-dampening beach. The wave-dampening system and beach allow taking measurements with a 12 minute intervals between carriage runs. Forces and moment were measured with an Izumi six-component strain-gage type load cell and six-channel Izumi amplifiers for signal conditioning. The measurement of the far-field wave elevations was performed using a longitudinal wave-cut method with a servo type wave measurement system. TPIV was used to acquire the 3D velocity field. TPIV is an extended concept of stereoscopic PIV, which enables measurements of all three velocity components in a $3 \mathrm{D}$ measurement volume. The volume measurement is based on volume reconstructions of the particle images from multiple cameras viewing from different angles.

The experimental grid resolution is about $1 \mathrm{~mm}$ in all directions, i.e., $\Delta_{\mathrm{EFD}}=0.00057 L_{P P}$, and the data rate is $13 \mathrm{~Hz}$. The averaged flow field data were obtained by ensemble averaging for ten repeat runs for each drift angle. Each run was for 20 seconds, thus the total averaging sample was 2,600.

The experiments were performed in calm-water condition at three static drift angles $\beta=0,10$ and 20 degrees at $F r=0.28$. This $F r$ corresponds to the full-scale cruising speed of the vessel at 20 knots and corresponds to $1.53 \mathrm{~m} / \mathrm{s}$ for model scale. The corresponding model scale is $R e=4.65 \times 10^{6}$, based on a water temperature of $20^{\circ} \mathrm{C}$. The model was fixed at the dynamic sinkage $\sigma=1.92 \times 10^{-3} L_{P P}$ and trim $\tau=-0.136$ degree (positive bow up) for all drift angles. The sinkage and trim values were based on the experimental values obtained by Longo and Stern (2005) for bare hull 5415 at $\beta=0$ and $F r=0.28$. In order to initiate transition to turbulence, cylindrical studs were placed in a row at $x / L_{P P}=0.05$ on the hull. The studs have a height of $1.6 \mathrm{~mm}$, a diameter of $3.2 \mathrm{~mm}$ and were fixed with a spacing of $9.5 \mathrm{~mm}$. The dimensions and placement of the studs are in accordance with the recommendations of the ITTC (2002).

Herein, the data procured for $\beta=0$ and $\beta=20$ conditions are used. The data includes: forces and moments; wave elevation and profiles; vortical structures and axial and cross-plane variations of flow variables in the vortex core; contour of flow variables at ten planes along the hull, i.e., $x / L_{P P}=0.06,0.1,0.12,0.2,0.3,0.4,0.6$, $0.8,0.935$ and 1.0; and iso-surfaces of flow variables at selected $3 \mathrm{D}$ volumes along the primary vortices, i.e., at $x / L_{P P}=0.06,0.1$, $0.12,0.2,0.3,0.4$ and 0.6 for $\beta=0$, and $\mathrm{x} / L_{P P}=0.06,0.1,0.12,0.2$, $0.4,0.6,0.8$ and 0.935 for $\beta=20$. The $3 \mathrm{D}$ volume measurements included the three velocity components which allowed calculation of velocity derivatives, thereby allowing visualization of vortical structures. A preliminary unsteady analysis is performed for the sonar dome vortex for $\beta=20$. The frequency content of the unsteadiness of the vortex was analyzed using velocity time histories at five points across the vortex core at $x / L_{P P}=0.4$ (Egeberg et al., 2014).

Experimental uncertainty for the axial force, side force and yaw moment is $U_{D}=3.6 \% \mathrm{D}, 5.4 \% \mathrm{D}$ and $2.6 \% \mathrm{D}$, respectively. Uniform flow tests were conducted to assess the accuracy of the TPIV measurements. The averaged uncertainty in the procurement of the velocity components is $U_{D}=2.5 \% D$ and for free-surface is $U_{D}=5 \% D$. The averaged uncertainty in the prediction of the derivative variables is $U_{D}=8.0 \% D$ for $\beta=0$ and $U_{D}=6.8 \% D$ for $\beta=20$.

\section{CFD Computations}

The submissions for $\beta=0$ and 20 included: CFDShip-Iowa results from IIHR; ReFRESCO results from MARIN; ISIS-CFD results from ECN/CNRS; NavyFOAM results from NSWCCD; and STAR-CCM+ results from NTUA. In addition, STAR-CCM+ results from FORCE were submitted for $\beta=20$. Table 1 list the main characteristics of the solvers, simulations and submitted results. The following summarizes the numerical methods and model, and simulation setup used by the solvers.

CFDShip-Iowa is a finite-difference solver (Huang et al., 2008) which uses multiblock overset structured grids and level-set method for free-surface modeling. The simulations were performed using hybrid $2^{\text {nd }} / 4^{\text {th }}$ order convection scheme using near-wall anisotropic URANS and DES turbulence models, which were identified to be most accurate in G2010 (Bhushan et al., 2014). Simulations were performed with $10 \mathrm{M}, 48 \mathrm{M}$ and $84 \mathrm{M}$ grids for $\beta=0$, and $10 \mathrm{M}, 48 \mathrm{M}, 84 \mathrm{M}$ and $250 \mathrm{M}$ grids for the $\beta=20$. The grids included overset grid blocks to refine the grids to better resolve the vortices all along the hull. For the $\beta=0$, the finest grid resolution was $0.00122 L_{P P}$, which is two times coarser $\Delta_{\text {EFD. }}$ The finest grid contained 7-8 grid points within the primary vortex 
core, at the location when the vortex separates from the surface. For $\beta=20$, the finest grid was the adapted $84 \mathrm{M}$ grid which has a resolution was $0.00122 L_{P P}$, which is two times coarser than $\Delta_{\text {EFD. }}$ The finest grid contained 14-15 grid points within the primary vortex cores. For $\beta=0$, URANS results on $84 \mathrm{M}$ grid provided the best prediction of the boundary layer and TKE at the nominal wake plane compared to DES, and was selected as the best result. For $\beta=20$, DES results on the finest $84 \mathrm{M}$ grid provided significantly better predictions than URANS, and was selected as the best result.

ISIS-CFD is a finite volume solver (Wackers et al., 2012). The simulations were performed using $2^{\text {nd }}$ order schemes using nearwall isotropic and anisotropic URANS and DES turbulence models and VoF method for free-surface modeling on hexahedra unstructured grids. The grids used for the $\beta=0$ consisted of $22 \mathrm{M}$ and $81 \mathrm{M}$ cells. The grid included local refinement better resolve the progression of the vortices up to $\mathrm{x} / \mathrm{L}_{\mathrm{PP}}=0.6$. The finest grid resolution was $0.00033 \mathrm{~L}_{\mathrm{PP}}$, which is finer than $\Delta_{\mathrm{EFD}}$. The finest grid included 10 grid points in the vortex core. The grids used for the $\beta=20$ consisted of: $27 \mathrm{M}$ grid, and adaptively refined grid with $10 \mathrm{M}$ cells $(10 \mathrm{M}+\mathrm{AGR})$. The adaptive grid refinement was limited only to the early part of the progression of the vortex. For $\beta=0$, it was reported that the results improved with grid refinement, and anisotropic URANS model, EARSM, provided better results than the isotropic counterpart. DES predicted too intense vortices due to a lack of TKE in the core of the vortex. Thus, results using EARSM URANS on $81 \mathrm{M}$ grid was identified as the best. For $\beta=20$, the results obtained on the coarse but locally refined $10 \mathrm{M}+\mathrm{AGR}$ grid were better than those obtained on a seemingly finer grid $(27 \mathrm{M})$ with no automatic local grid refinement. Thus, results using EARSM on 10M + AGR was identified to be the best.

ReFRESCO is a finite volume solver (Vaz et al., 2009). The simulations were performed using $2^{\text {nd }}$ order schemes using nearwall isotropic RANS turbulence models. For $\beta=0$, SST1994, SST2003 and KSKL RANS models were used. A separate simulation using VoF method with a $37 \mathrm{M}$ cells unstructured hexahedra-dominant grid was performed to obtain the free-surface deformation. Subsequently, the free-surface elevation was used as the top boundary for RANS computations using multiblock structured grids consisting of $1.3 \mathrm{M}$ to $45 \mathrm{M}$ cells, to obtain the flow details beneath the water surface. For $\beta=20$, double body simulations were performed using near-wall isotropic SST1994 and SST2003 RANS models on 8 different multiblock structured grids consisting of $1.3 \mathrm{M}$ to $33.6 \mathrm{M}$ cells. The quantitative grid resolutions were not discussed, however, a qualitative assessment of the grid distribution at different planes shows that the finest grid has intermediate resolution among the computations. For both the cases, results improved with grid refinement. In addition, the SST1994, SST2003 and KSKL predictions were similar for the onset of vortices. However, for the progression of the vortices SST2003 performs slightly better than others. For $\beta=0$ and 20 results using SST2003 RANS on 45M and $34 \mathrm{M}$ grid, respectively, were identified to be the best.
NavyFOAM is a finite volume solver (Kim et al., 2014). The simulations were performed using $2^{\text {nd }}$ order schemes using isotropic $\mathrm{k}-\omega$ turbulence model with wall-function (WF) boundary condition and VoF method for free-surface modeling, on hexahedra unstructured grids. They authors reported that they obtained insignificant differences between linear k- $\omega$ models and their EARSM versions for the resolution of vortices, thus isotropic models were used for the study. The grids used for $\beta=0$ consisted of 9M, 24M and 51M cells with $y^{+}=60$ for half domain. Some initial tests were performed using anisotropic URANS models, which are not part of the study, but are discussed to emphasize the effect of turbulence model anisotropy. The grids used for $\beta=20$ consisted of $18 \mathrm{M}, 20 \mathrm{M}$ and $68 \mathrm{M}$ cells with $y^{+}=$ 60 . The $20 \mathrm{M}$ grid did not include the effect of side-walls, whereas both $18 \mathrm{M}$ and $68 \mathrm{M}$ grids included the effect of side walls. The quantitative grid resolutions were not discussed. However a qualitative assessment shows that the finest grid for the $\beta=0$ is well refined all along the hull, and the resolution is similar to that in CFDShip-Iowa and ISIS-CFD grids. The finest grid used for $\beta$ $=20$ is well refined all along primary vortex cores, and the resolution is similar to that in CFDShip-Iowa grids. The results submitted from the simulations included: forces and moments; wave-elevations and profiles; 3D mean vortical structures planar plots; and limited volume plots. It was reported that results improved with grid refinement for both the cases. For $\beta=0$, the results obtained using $\mathrm{BKW}$ on $51 \mathrm{M}$ half domain grid were identified to be the best. For $\beta=20$, results obtained using BKW on $68 \mathrm{M}$ grid with side-walls were identified to be the best.

STAR-CCM+ from CD-adapco is a finite volume solver, which was used by both NTUA and FORCE groups. Both the groups used $2^{\text {nd }}$ order schemes, isotropic URANS turbulence with WF boundary condition and VoF free-surface models on hexahedra unstructured grids.

NTUA used k- $\varepsilon$ URANS turbulence for both $\beta=0$ and 20 . The grids used for $\beta=0$ included $2.5 \mathrm{M}, 3 \mathrm{M}, 3.5 \mathrm{M}$ and $4.4 \mathrm{M}$ cells with $\mathrm{y}^{+} \sim 30-40$. The grids used for $\beta=20$ included $4.6 \mathrm{M}, 5.7 \mathrm{M}$ and $7 \mathrm{M}$ cells with $\mathrm{y}^{+} \sim 30-40$. The grid were refined by cutting down the size of cells by $50 \%$ in each simulation in the area close to the hull, and placing the majority of the cells close to the freesurface and bilge keels. The quantitative grid resolutions were not discussed, however, a qualitative assessment shows that the grids were coarsest among the computations for both cases. Since, the grid refinement was mostly performed near the bilge keel and free-surface, thus the changes in the solution were not significant on grid refinement. Turbulence modeling sensitivity study was not performed. The best results were identified to be those predicted using $\mathrm{k}-\varepsilon$ URANS with $3 \mathrm{M}$ and $5.7 \mathrm{M}$ grids for $\beta=0$ and 20 , respectively.

FORCE used SST URANS turbulence for $\beta=20$. The grid consisted of $7 \mathrm{M}$ cells with $y^{+}=15$. The quantitative grid resolutions were not discussed, however a qualitatively assessment shows that the grid is coarser than other computations, but finer than STAR-CCM+ (NTUA) computation. Grid and/or turbulence modeling sensitivity study was not performed. 
Most of the submissions performed simulations on multiple grids to assess the grid sensitivity of results and/or to reduce numerical uncertainty. The grid refinement was performed manually by adding refinement blocks along the vortex core in CFDShip-Iowa and NavyFOAM. Whereas, ISIS-CFD used an automatic grid refinement strategy using local Hessian of pressure. Considering the grid resolution in the vortex core, for $\beta=0$ ISIS-CFD, CFDShip-Iowa and NavyFOAM used similar grid resolution, followed by ReFRESCO, and NTUA used the coarsest grid. For $\beta$ $=20$, both CFDShip-Iowa and NavyFOAM used similar grid resolution, followed by ReFRESCO and ISIS-CFD. Both NTUA and FORCE used significantly coarse grids. Numerical uncertainty estimates were reported only by CFDShip-Iowa and ReFRESCO. CFDShip-Iowa reported URANS involved numerical uncertainty $U_{S N}=2.2 \% \mathrm{~S}$ and $14 \% \mathrm{~S}$ for integral quantities and local variables, respectively, where $\mathrm{S}$ is the CFD simulation result. DES involved $U_{S N}=1.25 \% \mathrm{~S}$ and $16 \% \mathrm{~S}$ for integral quantities and local variables. ReFRESCO reported $U_{S N}=$ $1.7 \%$ for integral quantities.

The detailed results from each institute on all the grids have been presented in Bhushan et al. (2015). The validation study focuses mostly on the best results picked by each institute from their solver. However, the discussions are based on all simulations to explicate the effects of grids, turbulence models, and free-surface. Note that the solvers used in the study have been rigorously validated in the past for simple benchmark cases, and published in the open literature, before being used for ship hydrodynamics applications. Readers are referred to the references for each solver for details. Thus, it is expected that variability in the predictions due to difference in model implementation is insignificant compared to variability due to: solution approach (finite difference vs volume), numerical scheme, modelling approach (URANS vs DES, level-set vs VoF) and grid resolution, which are assessed herein.

\section{Assessment and Validation Approach}

The forces and moments are presented in Newtons $(\mathrm{N})$ and Newton-meter $(\mathrm{N}-\mathrm{m})$, respectively. The local variables are presented in non-dimensional form. The $\mathrm{x}, \mathrm{y}, \mathrm{z}$ locations and wave elevation are non-dimensionalized based on hull length $\mathrm{L}_{\mathrm{PP}}$; the velocities using free-stream velocity $\mathrm{U}_{0}$; vorticity using $\mathrm{U}_{0} / \mathrm{L}_{\mathrm{PP}}$, and TKE using $\mathrm{U}_{0}^{2}$.

The overall verification and validation approach mostly followed Stern et al. (2001) but using either the Xing and Stern (2010) factor of safety or Eça and Hoekstra (2014) least-square verification methods. The numerical uncertainties $\left(U_{S N}\right)$ are computed using the root-sum-square of the iterative $\left(U_{I}\right)$, grid $\left(U_{G}\right)$ and time step $\left(U_{T}\right)$ uncertainties following Stern et al. (2001) as below:

$$
U_{S N}=\sqrt{U_{G}{ }^{2}+U_{T}{ }^{2}+U_{I}{ }^{2}} \% S
$$

$S$ is the CFD solution on the finest grid. However, note that other $\mathrm{V} \& \mathrm{~V}$ approaches use a different formula for the numerical uncertainty quantification. For example, ASME (2009) recommends a linear addition of $U_{I}$ with RMS of $U_{G}$ and $U_{T}$.

The comparison error $(E)$ is used as quantitative metric of validation, which is computed as:

$$
E=\frac{(S-D)}{D} \times 100
$$

where $D$ is the experimental values. Note that the $\%$ errors are expressed in terms of $D$, which are referred to as $\% D$. Herein only the absolute value of the errors are reported. For parameters with multiple errors, such as those for the vortex core or waveelevation profiles or the entire CFD submission set, an averaged error $\bar{E}$ is reported, which is:

$$
\bar{E}=\sum_{i=1}^{N}\left|E_{N}\right| \% D
$$

where, $N$ is either the number of points considered on the profiles or the number of submissions. $\left|E_{N}\right|$ is the absolute value of the error at an individual point on the profile. The standard deviation of the errors is computed as:

$$
S D=\sqrt{\frac{1}{N} \sum_{i=1}^{N}\left(\bar{E}-E_{i}\right)^{2}} \% D
$$

The validation is performed by comparing the errors with the validation interval $\left(U_{V}\right) . U_{V}$ is computed using the averaged numerical uncertainty $U_{S N}$ values reported for the solvers and experimental uncertainty $\left(U_{D}\right)$ as,

$$
U_{V}=\sqrt{U_{S N}{ }^{2}+U_{D}{ }^{2}} \% D
$$

The CFD predictions are validated at an interval $U_{V}$ if

$$
\text { | } E \mid \% D \text { or } \bar{E} \% D \leq U_{V} \% D
$$

If the errors are larger than the validation interval, then the predictions are not validated.

For the predictions of forces and moment, errors are reported for each computation, and the mean and standard deviation of the errors for all the computations. The validation interval for the integral quantities is computed to be $U_{V}=1.83 \% D$.

The wave elevation pattern and profiles are compared with experimental data both quantitatively and qualitatively. Numerical uncertainties are not available.

The vortical structures are visualized using the iso-surface of Qcriteria $(Q=50)$ and $\lambda_{2}$-criterion. Onset and separation inception of the primary vortices are discussed for steady vortices for $\beta=$ 0 , and mean and instantaneous vortices for $\beta=20$. The onset of the vortex is identified by tracking the $\mathrm{Q}$ isosurfaces up to the hull surface. The inception of the vortex separation and the separation type are identified from the surface streamline pattern following Surana et al. (2006) study. The study identified that three-dimensional steady separation or reattachment can be categorized as: (1) closed separation; (2) open separation; and (3) open-closed-separation. The 3D flow separation starts from 
the body surface, and the separation pattern can be identified from the skin friction pattern. Closed separations are identified by the presence of saddle points, foci and nodes in the surface streamline (or skin friction) pattern. Open separation, also known as cross-flow separation, is identified by the converging surface streamline patterns. Open-closed separations are identified when the surface streamline pattern shows converging streamlines originating from a saddle point, but do not terminate in a node.

The progression of the vortex is validated by comparing the axial and cross-plane distribution of the flow variables (location, $Q$ values, vorticity components, axial velocity and TKE) in the core. The core is identified as the location of peak $Q$ value. The size of the vortex is defined based on the $Q=10$ iso-surface for $\beta=0$ and $Q=100$ iso-surface for $\beta=20$. The validation variables included vortex core location, strength, streamwise velocity and TKE at different axial locations with experimental data. Errors are reported for the primary flow variables for each solver, as well as averaged error, standard deviation and axial variation of the errors using all the computations. The validation interval for the vortex core predictions is computed to be $U_{V}=$ $16 \% D$ and $18.6 \% D$ for $\beta=0$ and 20 , respectively.

The planar plot prediction were mostly used to validate the overall flow structures including the interaction of vortices with each other and with the hull boundary layer, as they include narrow contour scales. The volume plot provided a quantitative validation of the vortex strength and qualitative validation of the $3 \mathrm{D}$ flow structures. The flow variable along and across the vortex were extracted from planar and volume data/results for quantitative validation.

The unsteadiness of the vortices was analyzed for $\beta=20$ at $\mathrm{x} / \mathrm{L}_{\mathrm{PP}}$ $=0.4$. The analysis was performed using fast Fourier transform (FFT) of the velocity time history in the vortex core. CFDShipIowa results also analyzed resolved TKE budget for $\beta=20$.

\section{FORCES/MOMENTS AND WAVE ELEVATION}

\section{Straight-ahead $\beta=0$}

As shown in Table 2, CFDShip-Iowa ReFRESCO and STAR$\mathrm{CCM}+$ resistance predictions compare within $2.2 \%$ of the data, whereas NavyFOAM and ISIS-CFD predictions convergence of a value lower than that of the experiments, and show $E=5.7 \%$ and $6.2 \%$, respectively. The averaged resistance predictions compared with $3 \%$ of the experimental data, and error standard deviation were $1.7 \%$. The averaged error $E=3 \% \mathrm{D}<U_{V}=4 \% \mathrm{D}$, thus the CFD computations are validated.

The under prediction by both ISIS-CFD and NavyFOAM are unexpected as they used finest grids among computations. NavyFOAM group pointed out that the differences between CFD and experiments could be due to the negligence of additional drag from the studs, which are used to trigger turbulence in the experiment, in the simulations. However, this explanation is not supported by: (a) grid studies performed by CFDShip-Iowa, REFRESCO and STAR-CCM+, which converge to the data; (b) previous experimental studies, which showed that resistance measured from different facilities with different turbulence trigger mechanisms converge to same value; and (c) G2010 study wherein CFD results converged to data. The ISIS-CFD and NavyFOAM groups should further investigate this issue using both intermediate and finer grids, and near-wall grids.

STAR-CCM+ predictions using WF were comparable to CFDShip-Iowa and ReFRESCO predictions using near-wall turbulence model. Similarly, NavyFOAM predictions using WF were comparable to ISIS-CFD predictions using near-wall turbulence model. This suggests that WFs with sufficiently fine near-wall grid resolution $y^{+} \leq 60$ have no significant penalty for resistance predictions.

As shown in Fig. 2, CFD wave-elevation predictions are comparable and compare within $3.8 \% D$ of the data. The errors are smaller than $U_{D}$, thus CFD predictions are validated, but validation interval cannot be defined. The predictions do not show dependence on turbulence modeling, as concluded on G2010. ReFRESCO performs the best for the prediction of wave elevation away from the hull. This is probably due to the use of a finer grid than other solvers for free-surface resolution. CFDShip-Iowa wave elevation predictions using level-set, both near and away from the hull, are very similar to other computations using $\mathrm{VoF}$.

\section{Static Drift $\beta=20$}

As shown in Table 3, the axial forces were predicted within $4.5 \%$ of the experiments by CFDShip-Iowa, ISIS-CFD, NavyFOAM and STAR-CCM+ (NTUA). STAR-CCM+ (FORCE) predicted larger error of $13 \%$. ReFRESCO predicted the largest error of $20 \%$. The side force predictions compared within $4.6 \%$ of the experiments by most solvers, except for $6.5 \%$ error reported by NavyFOAM and $16.6 \%$ reported by ReFRESCO. The averaged yaw moment predictions compared within $3.4 \%$ of the data. The lowest error of $0.25 \%$ were predicted by CFDShip-Iowa, STAR-CCM+ (FORCE) and NavyFOAM reported the largest error of $5.4 \%$ and $7 \%$, respectively. The largest errors in ReFRESCO are due to the use of double-body. The larger errors in STAR-CCM+ (FORCE) axial force predictions could be due to coarse grid resolution, however surprisingly STAR-CCM+ (NTUA) reported significantly better results for a similar grid size. The averaged errors (excluding double body results) for forces and moment is $<3.7 \% D$, and the standard deviation of the errors are less than $3 \%$. The averaged forces and moment predictions shows $E=$ $3.7 \% D \leq U_{V}=4.23 \% D$, thus they are validated.

CFDShip-Iowa and ISIS-CFD predictions using near-wall turbulence model are more accurate for $\mathrm{X}$ and $\mathrm{N}$ than STAR$\mathrm{CCM}+$ (FORCE) and NavyFOAM prediction using WF. This suggests that the use of WF has a penalty for forces and moment predictions, even if near-wall grid resolution $y^{+} \leq 60$. Surprising STAR-CCM+ (NTUA) WF simulations shows resistance and moment predictions comparable to near-wall predictions. Thus, considering the STAR-CCM+ (NTUA) predictions and a wide 
range of grids used in the study, no concrete conclusion can be drawn regarding the accuracy of WF.

NavyFOAM predictions with and without sidewalls on coarse grid showed that blockage does not significantly affect axial force and yaw moment predictions. However, the inclusion of sidewalls increase the side force by $6 \%$. A good agreement of NavyFOAM's axial force predictions with other CFD codes, also confirm that blockage does not affect axial force predictions. Considering a reasonable prediction of side force by other solvers without the sidewalls, no concrete conclusions can be drawn regarding the effect of blockage on side force. Further research is required to investigate the effect of blockage on side force and yaw moment predictions.

The experimental mean wave elevation in Fig. 3 shows an asymmetric pattern with $\alpha=10$ and 60 degrees with respect to the hull axis on windward and leeward sides, respectively. Wave elevation plots are available only from CFDShip-Iowa, ISIS-CFD and NavyFOAM. These solvers overall predict the asymmetric pattern well. ISIS-CFD performs better than CFDShip-Iowa for the prediction of wave amplitudes away from the hull on the leeward side. The NavyFOAM predictions show the effect of wall interference away from the hull, and miss some details of the wave crests and troughs. The averaged error in the CFD predictions is $8.5 \% D$, which is larger than $U_{D}$. Similar to $\beta=0$, the wave elevation predictions do not show dependence on turbulence modeling.

\section{VORTEX STRUCTURES AND ONSET, SEPARATION AND PROGRESSION ANALYSIS}

\section{Straight-ahead $\beta=0$}

As shown in Fig. 4 and Table 4, the experimental data confirms the presence of primary sonar dome (SDV) and fore-body keel (FBKV) vortices, and secondary bilge keel (BKV) and sonar dome trailing edge (SDTEV) vortices in the flow, as identified in G2010. SDTEV is not visible from Q plots, but is identified from $x / L_{P P}=0.12$ slice. The other vortical structures expected for this case are aft-body keel vortex (ABKV) and transom vortices. ABKV is not clearly visible, as the vortices are dissipated in the aft-body. Transom vortices could not be validated, as measurements were not performed in the transom wake. The data shows that SDV decays by $x / L_{P P}=0.4$, but FBKV extends all along the hull.

All the simulations agree regarding the prediction of SDV, FBKV, BKV, SDTEV, ABKV and transom vortices. Among the URANS results, ABKV is most prominent in ReFRESCO results, and MHV is predicted only in CFDShip-Iowa and ISISCFD DES results. NavyFOAM and ISIS-CFD results predicted multiple vortices near the free-surface aligned with the bow Kelvin waves, which were not predicted in other simulations or observed in experiments.

CFDShip-Iowa predictions showed that SDV, FBKV, SDTEV and $\mathrm{MHV}$ are associated with converging streamline pattern, thus were identified as open-type separation. ISIS-CFD,
ReFRESCO and NavyFOAM results confirmed that SDV has open-type separation. ISIS-CFD results also confirmed that SDTEV has open-type separation. CFDShip-Iowa predictions were further analyzed to study the open-type (or cross-flow) separation mechanism. To achieve this, the pressure gradients along and across the converging streamlines, and the distribution of wall shear stress and its gradients were studied. Results for SDV as shown in Fig. 5. The study revealed that open-type separations are generated due to negative and positive cross-flow pressure gradients below and above the converging streamlines, respectively. The vortex separates from the surface due to the presence of adverse axial pressure gradient along converging streamlines. The vortex separation location is identified from the peak of $\operatorname{div}\left(\tau_{w}\right)$, consistent with Simpson et al. (1992, 1996) studies.

As shown in Table 2, CFDShip-Iowa predictions show that FBKV extend farther than SDV, consistent with the data. Whereas, other CFD predictions show that SDV extends farther than FBKV. The variation of the mean flow variables in the SDV and FBKV cores are compared with the experimental data in Fig. 6. The data shows that both the SDV and FBKV decay with their progression, whereas SDV decays much faster than FBKV. SDV moves towards the centerplane and downward, whereas FBKV moves away from the centerplane and towards the hull. The axial velocity recovery in the vortex core suggests that SDV interacts with the hull boundary layer only during the early stages, whereas FBKV interacts strongly with the boundary layer throughout. The turbulence level in the vortex core decays with progression. FBKV core shows higher turbulence intensity and slower decay than that in the SDV core.

The vortex core analysis was submitted only by CFDShip-Iowa, ISIS-CFD and ReFRESCO. For the SDV core, all submissions agree with one another for the prediction of axial velocity, but vary for the other variables, as shown in Table 5. Both ReFRESCO and ISIS-CFD over predict $\mathrm{Q}$ and under predict $\omega_{\mathrm{x}}$, whereas CFDShip-Iowa shows an opposite trend. The core location is predicted best by CFDShip-Iowa, and TKE is predicted better by ReFRESCO and ISIS-CFD. Note that the predictive capability for TKE and vortex strength are linked, such that the solvers that under predict TKE consequently over predict vortex strength. As shown in Fig. 6, in both ISIS-CFD and CFDShip-Iowa predictions, the errors increase with the progression, whereas the errors decrease with progression in ReFRESCO predictions.

For the FBKV core, submissions show wide variation in predictions for all the quantities. As shown in Table 5, the vortex strength is under predicted by all solvers; $\mathrm{U}_{\mathrm{x}}$ is over predicted for $x / L_{P P}>0.3$ in both CFDShip-Iowa and ReFRESCO, whereas it is predicted well by ISIS-CFD; and TKE is over predicted in both CFDShip-Iowa and ISIS-CFD, whereas it is under predicted by ReFRESCO. Core location is best predicted by ISIS-CFD. The errors increase with the progression for all the submissions.

The variation of flow variables in the SDV and FBKV core cross-plane was analyzed using CFDShip-Iowa as shown in Fig. 
7 at $x / L_{P P}=0.2$ and 0.3 . Both experimental data and CFD results show a Gaussian-type distribution of the axial velocity and $\mathrm{Q}$ with damping in the boundary layer. CFDShip-Iowa predicts that axial velocity and $\mathrm{Q}$ peaks are aligned for both vortices, whereas experiments show alignment only for FBKV. As shown in Table 6 , the best predictions are obtained for axial velocity and worst for TKE; and SDV predictions are better than those for FBKV.

Overall, the FBKV predictions errors are $20 \%$ lower than those for SDV. However, the SD in predictions are 20\% higher for FBKV than that for SDV. The errors increase with the progression of the vortices from $60 \%$ to $120 \%$ for SDV, and $20 \%$ to $60 \%$ for FBKV. The averaged errors for the vortex axial core and cross-plane predictions are $63 \% D$ and $43 \% D$, respectively. The best axial core predictions are obtained using CFDShip-Iowa URANS for which $\bar{E}=25.5 \% D$. The error is higher than the $U_{V}=15.8 \% D$, thus CFD predictions are not validated.

\section{Static Drift $\boldsymbol{\beta}=\mathbf{2 0}$}

As shown in Fig. 8 and Table 4, the experimental data confirms that the presence of sonar dome tip (SDTV), leeward fore-body keel (LW-FBKV), bilge keel (BKV) and aft-body keel (ABKV) vortices on the leeward side, and bilge keel tip (BKTV), windward fore-body keel (WW-FBKV) and windward freesurface (WW-FSV) vortices on the windward side; as reported by Bhushan et al. (2011).

URANS results predicted steady vortical structures, whereas DES predicted unsteady vortical structures. CFDShip-Iowa DES predicts small-scale turbulent structures with $>95 \%$ resolved turbulence levels, superimposed over the large-scale structures. ISIS-CFD DES results showed that the modeled turbulence were dominant up to $x / L_{P P}=0.4$, whereas resolved turbulence dominated further downstream.

All the computations, including both the URANS and averaged DES results, predicted the primary vortices observed in the experiment. CFDShip-Iowa simulations predicted additional separation bubble and vortex (LW-SDBV) from the leeward sonar dome surface as shown in Fig. 9(a). CFDShip-Iowa DES results also predicted secondary circulations from bilge keel vortices, and leeward side mid-hull vortex (LW-MHV).

Instantaneous CFDShip-Iowa DES results show all the primary mean vortices, and several unsteady vortices on the leeward sonar dome surface, and unsteady free-surface vortices on the windward side. ISIS-CFD predictions did not show leading edge sonar-dome reconnecting vortex or leeward sonar dome bubble vortex, as identified by CFDShip-Iowa. However, they identified the presence of unsteady separated flow on the leeward sonar dome surface, due to the interaction of separated flow on the sonar dome and unsteady wave breaking. The vortex showed spiraling streamlines, which showed periodic growth and shrinkage and correlated with the leeward breaking wave.

Onset and separation analysis was performed using CFDShipIowa results, which showed that SDTV, LW- \& WW-FBKV,
BKV, BKTV, ABKV and LW-MHV all show open type separation. ReFRESCO results confirmed that SDTV, FBKV and $A B K V$ show converging streamline patterns, as expected for open-type separation. As shown in Fig. 9(b), sonar dome separation bubble originates from a sink node at the leading edge, is encased by a saddle point, and terminates at 2 focus points. The vortex satisfies flow topological rules expected for a close-separation over an isolated surface. WW-FSV separates from a source node close to the leading edge and attaches at a saddle point on the free-surface. The vortex satisfies flow topological rules expected for a close-separation formed over a body intersecting a wall or free-surface (Zhang and Stern, 1996). LW-FSV originates from a saddle point underneath the free-surface, does not terminate on the surface and shows converging streamlines downstream. Thus it is identified as open-closed type separation. LW-SDBV originates from a saddle point, does not terminate on the surface, and shows diverging streamlines downstream. The flow pattern is in contrast to the open-closed separation pattern identified by Surana et al. (2006), which states that the streamlines emerging from saddle point should show converging streamline pattern or terminate at limit cycle. Further analysis of the unsteady flow behavior of the primary vortices, including instability mechanism at the onset are discussed below.

The mean flow predictions by CFDShip-Iowa, ISIS-CFD and ReFRESCO in the SDTV and BKTV cores during progression are compared with experimental data in Fig. 10. The data shows that SDTV is roughly 4 times stronger than BKTV, and decays much slower. In addition, SDTV strengthens after onset up to $x / L_{P P}=0.3$, and decays thereafter. On the other hand, BKTV decays immediately after onset.

As shown in Table 5, both CFDShip-Iowa and ISIS-CFD computations agree within $6 \%$ of the data for the vortex core locations. Whereas, ReFRESCO shows higher errors, probably due to the negligence of free-surface effect. CFDShip-Iowa provided the best results for the SDTV strength, wherein good agreement with experiment is obtained in the early part of the progression, however, the vortex strength is under predicted further downstream. ISIS-CFD under predicts the vortex strengths by an order of magnitude, and ReFRESCO predicts even larger errors. In addition, CFD predictions show high core axial velocity and significantly lower TKE in the SDTV core compared to experiments. BKTV strength was predicted better by ISIS-CFD compared to CFDShip-Iowa, whereas latter performed better for TKE.

The variation of flow variables in the SDTV and BKTV core cross-plane was analyzed using CFDShip-Iowa as shown in Fig. 11. For SDTV, the analysis was performed at $\mathrm{x} / \mathrm{L}_{\mathrm{PP}}=0.2,0.4$ and 0.8, whereas for BKTV analysis was performed only at $\mathrm{x} / \mathrm{L}_{\mathrm{PP}}=0.6$. Experimental data shows that SDTV flows have asymmetric profiles early on which develops into a symmetric profile downstream. The asymmetry in SDTV distribution is due to the presence of secondary peaks and troughs in the core. The BKTV core variables show symmetric profiles. For both the vortices, the symmetric profile shows Gaussian-type distribution. CFDShip-Iowa predicts almost symmetric profiles 
for both the vortices throughout. As shown in Table 6, the predictions errors are similar for both the vortices, with lowest errors for $\omega_{\mathrm{x}}$ and highest error for TKE.

Overall, the BKTV prediction errors are 15\% lower than those for SDTV. Notable is the BKTV core axial velocity predictions, which is an order of magnitude better than that for SDTV. The errors do not show significant variation with progression. The averaged error for the vortex axial core and cross-plane predictions are $42.7 \% \mathrm{D}$ and $45.7 \% \mathrm{D}$, respectively. The best axial core predictions are obtained using CFDShip-Iowa DES for which $\bar{E}=34 \% D$. The error is significantly higher than $U_{V}$ $=19.3 \% D$, thus CFD results are not validated.

\section{PLANAR AND VOLUME VARIABLES}

\section{Straight-ahead $\beta=0$}

CFD predictions agree generally well with the experiments up to about $x / L_{P P}=0.4$, except that the vortices are aligned along the centerline, whereas they move away from the centerline in the experiment. Further aft, most CFD results show a stronger SDV than the FBKV, which is contrary to the experiment. Only CFDShip-Iowa result captures the stronger FBKV than SDV. The simulations capture only one closed streamline at $x / L_{P P}=$ 0.4 , and fails to capture closed streamline at $x / L_{P P}=0.6$, which is in contrast to the data which shows two closed streamlines due to SDV and FBKV at $x / L_{P P}=0.4$, and a single closed streamline only due to FBKV at $x / L_{P P}=0.6$ (refer to Fig. 12). Near the stern (refer to Figs. 13 and 14 at nominal wake plane), the boundary layer develops in a dual hook shape, one due to the interaction of hull boundary layer with FBKV and other due to the interaction of BKV. This is captured by most computations in various degrees, depending on the grid density in the aft ship. NavyFOAM fails to predict the presence of BKV, the effect of $\mathrm{BKV}$ is more pronounced than experiments in CFDShip-Iowa, and is less pronounced in ISIS-CFD. The ReFRESCO and NavyFOAM computations show an additional set of ABKV developing at the aft ship. The other solvers do not predict these vortices, partly due to insufficient grid resolution. In the experiment, these vortices are not visible. It may be that the $\mathrm{ABKV}$ is too close to the hull surface to be accurately captured with PIV.

\section{Static Drift $\beta=20$}

CFD predictions compare fairly well with the data, reproducing the major features of the flow, namely the characteristic vortices found in the data. The agreement between the predictions and the data is especially good during the onset and early part of the progression of the vortices, i.e., $x / L_{P P} \leq 0.2$. Fine details of the flow are harder to predict further downstream. In this respect, some results show better agreement with the experiments than others. URANS tends to cause the vortices to decay too fast, as demonstrated in Fig. 15. Furthermore, all URANS computations grossly fail to pick the high level of TKE in the vicinity of the vortex cores, severely under predicting the TKE level. DES predictions are better than URANS for the progression of the vortices. As they are able to predict the high TKE values in the vortex core (e.g., refer to Figs. 16 and 17) as expected, and predicts stronger vortex strength than URANS. However, the TKE production close to the hull surface appears to be over predicted by DES. In the CFDShip-Iowa results, the strength of the separated vortices and associated TKE in the cores of these vortices compare well the measurements, but vortices and TKE production close to the hull surface appear to be over predicted. Note that ISIS-CFD, with a mere 10M-element grid with AMR, does a commendable job in reproducing the salient features of the flow. Results from STAR-CCM+ (NTUA) on the coarsest 5.7M grid provide the least accurate result. The ReFRESCO results predict stronger vortex strength than other CFD solvers. This is due to the negligence of free-surface, which is expected to decrease vortex strength as concluded in G2010. The ReFRESCO results also show severely under predicted levels of TKE at the vortex cores, which was attributed to the use of the Dacles-Mariani correction for longitudinal vorticity. In addition, most of the submissions show high TKE near the free-surface, which is not confirmed by the data due to insufficient flow resolution. More study is required to find the origin of this discrepancy.

\section{GRID RESOLUTION AND TURBULENCE MODEL}

\section{Straight-ahead $\beta=0$}

The most important aspect of the grid refinement is the local grid characteristics in the flow regions where the vortical structures appear and progress.

Egolf et al. (2000) claimed that a minimum of 14 grid points across the vortex core and a $5^{\text {th }}$ order accurate scheme is required for "minimal diffusion". Considering this criterion, the data are sufficiently fine which resolves SDV and FBKV using averaged 17 to 20 points. None of the grids built by the contributors meet these strict requirements, but ISIS-CFD and CFDShip-Iowa grids are not so far from satisfying these conditions. CFDShip-Iowa grids have about 8 to 10 cells in the vortex core, as shown in Table 1. Although not shown, NavyFOAM's finest grid resolution is expected to be similar to CFDShip-Iowa and ISIS-CFD.

Computations show that it is essential to use grids with a sufficiently fine resolution at the sonar dome to accurately capture the onset of the vortical structures. Note that the FBKV and SDTEV could be captured only on extremely fine grids. With insufficient grid resolution, unduly large numerical dissipation leads to failure to resolve fine features of the flow and to correctly capture the topology and the number of vortices. This implies that it is essential to refine the grids in the vortex onset region, to which one does not pay attention most of the time. Although the sonar dome shape is not usual, one can wonder if this situation cannot be encountered for commercial ships with large protruding bow shapes, resulting from an optimization based on inviscid flow solvers.

In the near wake of the sonar dome, the flow appears to be mostly pressure-driven. The vortices progress in the flow along 
the hull, and the numerical dissipation is the largest influencing factor. This means that the grid should be adapted to the trajectories of the vortices to avoid any premature dissipation of the vorticity. This pleads in favor of the introduction of some local mesh adaptation if we want the grids to be suited to the flow characteristics of interest without being obliged to deal with meshes comprised of several hundreds of million points. In CFDShip-Iowa simulations, this is achieved with the use of the overset methodology (where an a priori adaptation is easily made once the locations of the most prominent structures are known). In ISIS-CFD simulations, this is achieved by the use of an automatic grid refinement strategy using local Hessian of pressure. NavyFOAM also made use of locally embedded blocks of fine grids. Most of the unstructured grid based solvers (e.g., STAR-CCM+) can be used with locally adapted grids.

However, as soon as the numerical dissipation is under control, the prediction depends strongly on turbulence closures. This leads to the second important conclusion. During the vortex progression, the turbulence modeling error is the major factor controlling the spatial evolution of the vortices far from the onset. The results provided by URANS models based on isotropic or anisotropic closures differ a lot from the predictions made by the hybrid RANS/LES closures as used by CFDShipIowa and ISIS-CFD.

At first glance, DES and its variants seem to predict vortical structures, which are richer and stronger than those predicted by URANS. But axial and cross-plane vortex core predictions in Tables 5 and 6 show that DES predictions show 3 to 4 times larger errors compared to URANS, primarily due to over prediction of vortex strength and under prediction of TKE.

The following general observations can be drawn from the submissions:

1. URANS computations based on isotropic turbulence models predict too weak longitudinal vortices, which are dissipated more quickly than that in the experiments. This means that the modeling error in URANS is quite large in the core of the vortices. However, the amount of TKE is reasonably well predicted up to $x / L_{P P}=0.6$, which seems to indicate that other turbulence quantities, such as turbulence transport or eddy viscosity are not correctly reproduced. From $x / L_{P P}=0.6$ to the stern, the predicted TKE is too high in the boundary layer between the core of the vortex and the hull, which most likely causes the premature decays of these vortices.

2. Effects of streamline curvature are significantly large in and around the core of the vortices. It has been long known that the isotropic closures are fundamentally not able to reproduce this physics. The anisotropic models seem to be marginally better. Differential Reynolds Stress models (DRSM) based on solutions of full Reynolds-stress transport equations can fundamentally account for effects of streamline curvature. Such an approach would probably improve predictions of the physics involved in the formation and evolution of vortices. One difficulty hampering use of the DRSM is its lack of robustness (compared to eddy-viscosity model) and higher computational cost for complex flows like the present ones.
Also, note that RANS is not well suited for unsteady flow, especially when the separated flows include large-scale turbulent coherent structures.

3. DES approaches look promising insofar as they predict more persistent longitudinal vortices compared to URANS. However, based on the experiments, the turbulence kinetic energy (TKE) in the core of the vortices is strongly underevaluated DES. This under-estimation of TKE leads to an under-estimation of the turbulent viscosity in the core of the longitudinal vortex, which results into a quasi-laminar core. This predicted laminarization of the flow in the core of the longitudinal vortex naturally explains the intensity and persistence over long distance of the computed vortical structures. Further, the laminarization of the flow is caused by modeled stress depletion in DES approach, which cannot be resolved by grid refinement.

\section{Static Drift $\beta=20$}

The grids are modest in size compared to those used for $\beta=0$. The experimental data resolves the primary vortex cores using about 27 - 33 points, whereas the finest grid as used by CFDShip-Iowa uses 15 to 25 points. The grid studies from all the solvers show that the URANS results improve with grid refinement. Only CFDShip-Iowa provides a systematic grid study using both modest and adaptively refined fine grids. The study shows that URANS results do not show significant improvement when the grid is refined from $48 \mathrm{M}$ to $84 \mathrm{M}$. Even on the finest grid, URANS predicts an excessive decay of the vortices soon after the separation, resulting in poor predictions compared to the experimental data. This behavior is also observed in other solvers. In addition, URANS grossly under predicts TKE levels in the vortex core, which is in contradicts the data. Thus, the errors in URANS predictions are more likely due to modeling issues rather than grid resolution.

DES results improve and the resolved TKE levels increase with grid refinement. DES results on the finest $84 \mathrm{M}$ grid provide $>95 \%$ resolved turbulence levels. The axial and crossplane vortex core predictions in Tables 5 and 6 show that DES predictions show on an average 15 to $20 \%$ lower errors compared to URANS, and the results improvements are significantly higher for downstream locations. However, DES over predicts and under predicts vortex strengths at onset and away from the hull, respectively. Considering the improvements in the results with grid refinement, the differences between CFD and experiments could be partly due to grid resolution. However, some of the differences are likely due to DES modeling deficiencies.

\section{INSTABILITY ANALYSIS AND TKE BUDGET}

Unsteady nature of the primary vortices and associated instabilities at onset, and TKE budget analysis were performed for $\beta=20$ simulations using CFDShip-Iowa DES. Note that this study was performed previously using un-adapted $10 \mathrm{M}, 48 \mathrm{M}$, and 250M grids, and not published elsewhere. 
Both URANS and DES predicted that SDTV and BKTV for 5415 at $\beta=20$ exhibit open-type cross-flow separation with spiral streamlines/vortices emanating from the hull. As the vortices progress downstream, the vortex core variable trends globally show axial velocity and vorticity decreases, pressure increases, and TKE remains nearly constant, as shown in Fig. 10 . The swirl ratio $\mathrm{S}=U_{\theta} / U_{x}$, where $U_{\theta}$ is the tangential velocity and $U_{x}$ is the axial velocity, at the onset of the instability shows high values of 0.76 to 0.82 , and decreases further downstream. DES predicts that the $\mathrm{St}=\mathrm{fL} \mathrm{LP}_{\mathrm{P}} / \mathrm{U}_{0}$ associated with the instability decreases with the progression of the vortex as shown in Fig. 18(a). Where, $\mathrm{f}$ is the dominant frequency along the vortex core evaluated using the pressure fluctuation, $\mathrm{U}_{0}$ is free-stream velocity and $L_{P P}$ is ship length. The scaling of the dominant frequency using the distance from the origin of spiral streamline location $(\mathrm{X})$ shows $\mathrm{St}_{\mathrm{X}}=\mathrm{fX} / \mathrm{U}_{0} \sim 0.75$ to 1.35 , as shown in Fig. 18(b).

For KVLCC2 at $\beta=30$ vortices, both URANS and DES predicted that the vortices exhibit open-type cross-flow separation from the hull. The vortices separate as a circular type, undergo helical mode instability, and transform into spiral vortices. Mostly, the vortex core axial velocity and vorticity showed a global decrease, whereas the core pressure and TKE showed a global increase. CFD predictions reported $\mathrm{S} \sim 0.46$ to 0.71 at the onset of the instability, and value decreased with the progression of the vortices. Instability analysis using DES shows that St decreases with the progression of the vortices, and $\mathrm{St}_{\mathrm{X}}=1.2 \sim 1.45$ as shown in Fig. 18(b).

Spiral vortices have been reported for swirling jets, wing tip vortices and delta wing leading edge vortices (Nelson and Pelletier, 2003; Gursul, 2009). In these cases, circular vortices exhibit vortex breakdown followed by helical mode instability and transformation into spiral vortices. The breakdown is characterized by a sudden expansion of the core and sharp gradients in core variables, i.e., drop in axial velocity and vorticity, and increase in pressure and TKE. The initiation of the vortex breakdown occurs if the swirl ratio is higher than the critical value $S_{c}$. For delta wings, $S_{c} \sim 1$ for a range of $R e$, as shown by Greenwell (2009). Further, Stx is constant along the core and ranges from 0.75 to 1.35 Gursul (1994).

Overall, the vortices for $5415 \beta=20$ and KVLCC $2 \beta=30$ have similarities with the swirling jet or delta wing vortices as they show helical mode instability, spiral streamlines/vortices, high swirl at instability inception, and $\mathrm{St}_{\mathrm{x}}$ range is consistent with those of delta-wings. However, the swirl ratio is lower and large differences are observed in the transition process from circular to spiral vortices. For swirling jet or delta wing vortices, the transition process is abrupt with a large change in vortex size and gradients of the vortex core variables. However, for ship flows, the transition process is abrupt but without large change in vortex size and gradients of core variables.

The dominant frequency for SDTV at $\mathrm{x} / L_{P P}=0.4$ is $2.66 \mathrm{~Hz}$, which compares within $11.3 \% \mathrm{D}$ of the experimental data (Egeberg et al., 2014). The leeward SDV separates from the sonar dome surface due to the merger of two shear layer instabilities into a Karman-like instability with $\mathrm{St}=0.132$. FBKV separation is identified due to shear-layer instability with $\mathrm{St}=0.0029$. The leeward sonar-dome separation bubble shows a flapping-like instability and the transom flow shows Karman-type shedding.

As shown in Fig. 19, the peak TKE is observed mostly on the high-speed side (identified by the concentrated streamlines) of the SDTV and BKTV cores, and close to the free-surface in the breaking wave regions. TKE budget shows that convectionproduction ratio $\mathrm{C} / \mathrm{P} \sim 0.6$. Turbulent transport $\mathrm{T} / \mathrm{P}$ is small $\sim 0.2$ on the $10 \mathrm{M}$ grid, and increases to 1.1 on the $250 \mathrm{M}$ grid. The pressure transport is higher on coarse grid PT/P 1 on the $10 \mathrm{M}$ grid and $\sim 0.75-0.8$ on $48 \mathrm{M}$ and $250 \mathrm{M}$ grids. Dissipation is lower than the production near the vortex inception, $\varepsilon / \mathrm{P} \sim 0.8$, and vice versa downstream, where $\varepsilon / \mathrm{P} \sim 2.1$. The transport terms are quite large compared to those reported for the canonical flows, whereas the convection term agrees with the values reported for those inside the separation bubble. The dissipation-production ratio is similar to those predicted for a surface piercing NACA 0024. Overall, results show that TKE is produced at the vortex inception and is transported along with the cores, as expected. The TKE is mainly transported occurs via pressure fluctuations on the coarse grid and turbulent fluctuations on the fine grid.

\section{DISCUSSIONS}

A detailed validation is performed to assess the predictive capability of the state-of-art CFD numerical methods and models, using concurrent experimental measurements, for flow over surface combatant 5415 at straight-ahead and static drift $\beta$ $=20$ degree. The test cases considered in the study cover a broad range of important features of the flow encountered in Navy's surface ships. The flow for the straight-ahead condition is characterized by vortices generated at the sonar dome and progressing in the vicinity of the hull boundary layer. Thus, the vortices are constantly fed by the turbulence created in the boundary layer. The static drift case is far more complex than the straight-ahead case. The flow features multiple vortices of various origins emanating along the hull, most notably from the sonar dome and the bilge keels, and unsteady breaking waves and ensuing bubbly mixture, and associated vortical structures progressing near the free-surface. Most of the vortices are displaced far from the hull once they are generated. Thus, the evolution of the mean flow and turbulence in their cores tend to occur in a free shear regime.

A valuable set of experimental data using TPIV has been procured for detailed validation of CFD predictions for flow over 5415 at $\beta=0$ and 20 . The validation data includes: integral force and moment, wave-elevation and profiles, and steady and unsteady local flow and turbulence planar and volume profiles, and for the first time vortical structures and their axial and cross-plane distribution during progression.

\section{Straight-ahead $\boldsymbol{\beta}=\mathbf{0}$}

CFD computations were from five different institutions on grid sizes ranging from $2.5 \mathrm{M}$ to $102 \mathrm{M}$ for the full domain. Most groups used at least one grid finer than $45 \mathrm{M}$. Thus, the grids 
used in the study are significantly finer than those used in G2010.

The averaged resistance predictions $E=3 \pm 1.7 \% D$. Compared to G2010, wherein $E=2.5 \pm 4.2 \% D$, the averaged error is slightly higher, but the standard deviation in the results is smaller. The averaged error is less than the validation interval $U_{V}=4 \% D$, thus entire computation set is validated. Results showed that WFs have no significant penalty for the resistance predictions due to sufficiently fine near-wall grid resolution, i.e., $y^{+} \leq 60$.

The wave elevation predictions compared within $3.8 \% D$ of the experiments. The predictions do not show dependence on turbulence modeling, as concluded on G2010 Workshop. Further, both level-set and VoF approaches provided similar wave elevation prediction both near and away from the hull.

All the computations agree well with the experimental data regarding the presence of primary vortices. DES predicted additional mid hull vortex, which was not observed in the experiment. Analysis of the results revealed that the vortices mostly exhibit open-type separation, which are generated due to negative and positive cross-flow pressure gradients below and above the converging streamlines, respectively. The vortex separated from the surface due to the presence of adverse axial pressure gradient along converging streamlines. The vortex separation location was identified from the peak of $\operatorname{div}\left(\tau_{w}\right)$, consistent with Simpson et al. (1992) study.

The CFD computations showed wide variation in the progression of the vortices, i.e., CFDShip-Iowa predicted that SDV decays faster than FBKV consistent with the experiments, whereas other computations predicted a faster decay of FBKV than SDV. All the solvers used similar hexahedra meshes, thus the difference between CFDShip-Iowa and other solvers could be due to the solution approach, i.e., use of finite difference solution approach compared to finite volume solution approach used by other solvers. The difference could also be due to the use of higher $4^{\text {th }}$-order upwind scheme by CFDShip-Iowa, compared to $2^{\text {nd }}$ order used by other solvers. Future work should focus on detailed TKE budget analysis and Reynolds averaged vorticity transport using both experiments and CFD, to elucidate the source of the discrepancy.

For the vortex core predictions, error increased with the progression of vortices. The core location and TKE predictions showed errors $<10 \% D$, whereas vortex strength predictions showed large errors of $125 \% D$. The axial and cross-plane vortex core predictions show averaged error of $53 \pm 50 \% D$. The best prediction for the axial core predictions was obtained using CFDShip-Iowa URANS, which predicted $\bar{E}=25.5 \% D$. Even the best prediction shows error higher than the validation interval of $16 \% D$. Thus, the results were not validated.

For the planar and volume variables, all the CFD predictions agreed well with the experiments up to about $x / L_{P P}=0.4$, but showed wide variations further downstream. In the downstream region, CFDShip-Iowa DES performed better than other solvers and URANS models for the prediction of axial vorticity, and predicted closed streamlines at mid-girth consistent with the experimental data. The solvers show varying degree of accuracy for the prediction of hook shape at the nominal wake plane. Some are over predictive and some under predictive, and no solver or model is identified to be better than other.

\section{Static Drift $\beta=20$}

CFD computations were from six institutions using grid sizes ranging from $4.6 \mathrm{M}$ to $250 \mathrm{M}$. Most of the grids used in the study are relatively coarse, compared to $\beta=0$, consisting of about $10 \mathrm{M}$ elements, except for those used by CFDShip-Iowa and NavyFOAM.

The averaged errors for forces and moment is $\leq 3.7 \pm 3 \% D$. The averaged errors are lower than the validation interval $U_{V}=$ $4.23 \% D$, thus the computational set is validated. Significant improvements are obtained for the prediction of integral quantities compared to the SIMMAN 2008 (Stern et al., 2011), as the errors in the axial force reduced by 10 points and that for yaw moment reduced by 5.5 points. Results seem to indicate that the use of near-wall turbulence model leads to more accurate axial force and yaw moment predictions compared to wall-functions. However, considering a wide range of grids used in the study, no concrete conclusion can be drawn. NavyFOAM results demonstrated that simulation without the wave basin wall blockage effect leads to higher errors for side force and yaw moment predictions. They concluded that further research is required to investigate the effect of blockage.

The wave elevation predictions compared reasonably well with the data close to the hull. Large errors were obtained away from the hull, which were attributed to coarse grid resolutions. The averaged prediction error is $8.5 \% D$. Both level-set and VoF approaches provided similar wave elevation prediction away from the hull, but showed differences in the elevation pattern close to the bow. In particular, VoF method predicted thin water sheet attached to the hull on finer grids, which is not confirmed by the data.

CFD results were in close agreement with each other for the prediction of the dominant vortical structures. But show large variation for the structures inside the leeward sonar dome separation bubble, and the free-surface vortices due to breaking waves. The vortex separations pattern are identified as either open-, closed- or open-closed type. The separation pattern and topology were consistent with those available in the literature. The closed-type separation satisfied the topological rules expected for a close-separation formed over an isolated body or body intersecting a wall or free-surface.

For the vortex core predictions, the errors were almost uniform throughout the progression. The lowest errors $\leq 5.3 \% \mathrm{D}$ were obtained for the location, and large errors up to $60 \% \mathrm{D}$ for vortex strength and axial velocity deficit. The axial and cross-plane vortex core predictions show averaged error of $44 \pm 24 \% D$. The best axial core predictions are obtained using CFDShip-Iowa DES, for which averaged $E=34 \% D$. Even the best prediction shows error higher than the validation interval of $19.3 \% D$. Thus, the results are not validated. 
For the planar and volume variables, all CFD predictions compared reasonably well with data during the early stage of the progression of the vortices, i.e., $x / L_{P P} \leq 0.2$. However, details of the flow are harder to predict further downstream and in this respect, some results show better agreement with the data than others. CFDShip-Iowa DES vortex strength and TKE predictions compared better with the measurements compared to other solvers. Among the URANS results, ISIS-CFD on 10M adaptive grid provides the best results, especially for TKE. STAR-CCM+ results on the coarsest $5.7 \mathrm{M}$ grid were excessively dissipative, and provided the least accurate result.

The primary vortices for $5415 \beta=20$ and KVLCC2 $\beta=30$ predicted by both URANS and DES show similarities with the swirling jet or delta wing vortices as they show helical mode instability, spiral streamlines/vortices, and high swirl at instability inception. In addition, $\mathrm{St}_{\mathrm{x}}$ range predicted by DES is consistent with those of delta-wings. However, the swirl ratio is lower and large differences in the transition process from circular to spiral vortices. For swirling jet or delta wing vortices, the transition process is abrupt with a large change in vortex size and gradients of the core variables, i.e., vortex breakdown. For ship flows, the onset/transition process is abrupt but without large change in vortex size and differences in gradients and trends of the core variables. Nonetheless, we retain the terminology vortex breakdown for this process. The differences between ship and aero flows are likely due to global geometry and pressure gradients and the interaction of the ship vortices with the boundary layer and other vortices.

The TKE budget showed that the peak TKE is obtained mostly on the high-speed side of the vortices. Turbulence is produced at the vortex inception and is transported along with the cores via pressure or turbulent fluctuations.

\section{CONCLUSIONS AND RECOMMENDATIONS}

The experimental data shows that the axial resistance obtained for $\beta=20$ is about 2 times higher than that for $\beta=0$. The primary vortices for $\beta=0$ are SDV and FBKV, and those for $\beta=$ 20 are SDTV and BKTV. FBKV and SDTV are the strongest vortices for $\beta=0$ and 20, respectively. All the vortices showed the highest strength close to the inception and decayed exponentially after progression. The SDTV strength is about 100 times higher and the decay rate is 3 times faster than FBKV. In both the cases, a momentum deficit is predicted in the vortex core, which increases with the progression of the vortex. The TKE value in the SDTV core is 60 times higher compared to that in FBKV core. For the former, TKE decays after the inception, whereas for the latter the TKE decays up to after-body and increases further downstream.

Limited studies reported numerical uncertainties due to grid resolution. The averaged $\mathrm{U}_{\mathrm{SN}}=1.7 \% \mathrm{~S}$ for forces and moment for both $\beta=0^{\circ}$ and $20^{\circ}$. The $\mathrm{U}_{\mathrm{SN}}=13.4 \% \mathrm{D}$ and $15.7 \% \mathrm{D}$ for $\beta=0^{\circ}$ and $20^{\circ}$, respectively, for the vortex core predictions. Detailed numerical uncertainty estimation of CFD solvers, including bias due to domain size, boundary conditions, time step size and other relevant user input parameters, are required to estimate the uncertainty of numerical simulations.

The forces and moments are predicted within $3 \% D$ of the data in both the cases, and the standard deviation in the CFD submissions is $<3 \% D$. The results are validated within $4.3 \% D$ confidence interval. The wave elevation predictions for $\beta=0$ and 20 showed averaged error of $3.8 \% \mathrm{D}$ and $8.5 \% \mathrm{D}$, respectively. The standard in the CFD submissions were $\leq$ $2.5 \% D$, in both the cases. The wave elevation pattern for $\beta=0^{\circ}$ were validated as errors $<U_{D}=5 \% D$. For the $\beta=0$ vortex core predictions, the averaged $E=63 \% D$ and standard deviation in $40 \% D$. For $\beta=20$ predictions, the averaged $E=44 \% D$ and standard deviation in $20 \% D$. The error distribution among the variables shows that, the errors for the core location are mostly $<$ $10 \% \mathrm{D}$ for both the cases. The core momentum deficit and TKE shows $20 \% D$ lower errors for $\beta=0$ compared to $\beta=20$. However, the vortex strength, $\mathrm{Q}$ and axial vorticity, are 50 to $70 \% D$ higher for $\beta=0$. The higher errors are primarily due to over prediction of SDV strength.

The study demonstrates that onset and separation of the primary vortices are predicted reasonably well even on coarse grids. But fine grids are required to capture the progression of the vortices, and secondary separations. The grid studies show that welladapted $50 \mathrm{M}$ or finer grids are required to obtain acceptable URANS predictions. The grids used for the $\beta=0$ by most groups meet this requirement. However, even finer grids are required to achieve the ideal resolution of 14 cells for vortex core resolution. For $\beta=20$, only CFDShip-Iowa and NavyFOAM computations use more than 50M cells, and other use significantly coarser grids. Hybrid RANS/LES simulations require much finer grids than URANS. For $\beta=20$, the DES predictions continue to improve, as the grid is refined to $84 \mathrm{M}$ nodes. Thus, even finer grids are required to obtain better results.

Most of the salient features of the flow are captured reasonably well by all participating codes, despite different choices of the grids and turbulence models. However, the accurate prediction of the finer details of the flow such as the secondary and tertiary vortices is much harder. Further studies using finer grids are required in order to minimize discretization error and to see if by doing that one can better resolve the fine details of the flow. With the current grids and solvers, there does not appear to be one turbulence model that can accurately predict all aspects of the flow for straight-ahead and drift conditions. DES is not able to fully resolve the TKE for the straight-ahead condition and under predicts the TKE levels. Nevertheless, DES appears to be the only model to accurately predict the TKE and vortex strength for separated vortices in $\beta=20$. In addition, most solvers over predict the vorticity and TKE near the free surface.

The study underlines two weaknesses of to-date high fidelity CFD tools: (i) the need of better grid design to control the numerical dissipation in the region where the onset of vortices takes place; and (ii) the need of more accurate turbulence closures to simulate the vortex flow dynamics. The following provides recommendations for the above two weaknesses. 
Solution-adaptive mesh refinement offers a cost-effective meshing strategy, which helps locally refine the grid along the vortex trajectory. However, the regularized Hessian of the pressure based refinement fails to refine the grid all along the vortex. Further research is required to identify refinement metric to track vortical structures. In this study, overset methodology or region-based adaptation is shown to be a viable alternative for solution-based grid refinement. However, overset methodology may include conservation and interpolation errors. Moreover, it cannot be used to refine in zones where vortical structures are not a priori expected, such as for unsteady flows. Therefore, its application is more limited than adaptive grid refinement.

In both the test cases, URANS predicted an excessive decay of the vortices, and resulted in large errors for the progression of the vortices. In addition, the URANS results from different solvers provided different decay pattern for the vortices for $\beta=0$. Further, they under predicted the levels of TKE in the vortex core for $\beta=20$. Considering the grid resolutions used in the study, the errors in URANS predictions are largely attributed to turbulence modeling errors, especially their inability to accurately account for the effects of streamline curvature and the misalignment of the Reynolds stresses and mean strain rate in the vortex core. Anisotropic URANS models show some improvement over the isotropic models for $\beta=0$. However, the differences between isotropic and anisotropic models are only marginal. It would be tempting to revisit the full RSTM closures, if their robustness can be improved on complex grids. However, it must be noted that URANS is not well suited for unsteady flow, especially when the separated flows include turbulent coherent structures.

DES predicts the levels of TKE and vorticity magnitude for $\beta=20$ with reasonable accuracy. However, it suffers from a major deficiency for $\beta=0$, where it predicts very low resolved turbulence levels. A similar conclusion was drawn in G2010 workshop, and it is identified that the deficiency is due to its inability to trigger resolved turbulence. For $\beta=20$, DES predictions improved with grid refinement and predicted over 95\% resolved turbulence levels on the finest grid. In addition, the flow predictions were better than those predicted by URANS. But, they predicted stronger vortex strength at the onset and weaker vortex strength downstream most likely due to modeled stress depletion issue.

LES are expected to provide more accurate CFD predictions than URANS, as they have less dependence on modeling; however, they are prohibitively expensive due to grid resolution requirements in the boundary layer. Hybrid RANS/LES models provide a reasonable alternative, wherein URANS is used in the boundary layer and LES in the wake. The research should particularly focus on investigation of: turbulence trigger models to enable transition from RANS to LES for slender body simulations; blended RANS-LES models as they include explicit LES modeling that are more rigorously validated than the LES mode of single parameter models, such as DES; and physics based RANS-LES blending rather than grid-based blending to address modeled stress depletion issues.
Future work should also focus on:

(a) Analysis of TKE and Reynolds averaged vorticity transport budgets both in experiments and CFD for $\beta=0$, can help understand the energy transfer from mean to turbulent flow (i.e., turbulence production) and eventual decay by molecular viscosity (i.e., dissipation). The difference in the production and dissipation pattern in SDV and FBKV can help understand the decay pattern of the vortices;

(b) More detailed analysis of the volume solutions to validate the predictions of vortical structures;

(c) Analysis of three-dimensional unsteady separation, vortex breakdown and scaling of instabilities during progression for $\beta=20 ;$ and

(d) In spite of the large effort and huge progress in going from PIV to TPIV, more experimental measurements are required for detailed validation, especially velocity and turbulence fields near the body and free-surface, and for unsteady vortex analysis.

\section{ACKNOWLEDGEMENTS}

The U.S. Office of Naval Research under Grant N00014-10-1-0017 sponsored the research at the University of Iowa under the administration of Drs. Thomas Fu and Ki-Han Kim. Drs. Patrick Purtell and $\mathrm{Ki}-\mathrm{Han} \mathrm{Kim}$ provided guidance to the international collaboration over the course of this study and Dr. Kim graciously aided in editing. Dr. Stephan Hitzel, Airbus, provided consultation on the assessment of the ship-flow vortex breakdown and helical mode instability/spiral vortices.

\section{REFERENCES}

ASME, "Standard for Verification and Validation in Computational Fluid Dynamics and Heat Transfer," V 20, 2009.

Abdel-Maksoud, M., Müller, V., Xing, T., Toxopeus, S., Stern, F., Petterson, K., Kim, S.-E., Eloot, K., Gietz, U., Schiller, P., Rung, T. Experimental and Numerical Investigations on Flow Characteristics of KVLCC2 at $30^{\circ}$ Drift Angle, $5^{\text {th }}$ World Maritime Technology Conference, November 3-7, Rhode Island, USA, 2015.

Bhushan, S., Carrica, P., Yang, J. and Stern, F. "Scalability and Validation Study for Large Scale Surface Combatant Computations Using CFDShip-Iowa," The International Journal of High Performance Computing Applications, Vo. 25, No. 4, 2011, pp. 466487.

Bhushan, S., Xing, T., Visonneau, M., Wackers, J., Deng, G., Stern, F. and Larsson, L. "Chapter 7: Post Workshop Computations and Analysis for KVLCC2 and 5415," Numerical Ship Hydrodynamics: An Assessment of the Gothenburg 2010 Workshop, Springer, 2014.

Bhushan, S., Yoon, H., Stern, F., Guilmineau, E., Visonneau, M., Toxopeus, S., Simonsen, C., Aram, S., Kim S.E. and Grigoropoulos, G. "CFD Validation for Surface Combatant 5415 Straight Ahead and Static Drift 20 Degree Conditions." IIHR Report No. 493, 2015.

Broglia, R., Aloisio, G., Falchi, M., Grizzi, S., Zaghi, S., Felli, M., Miozzi, M., Pereira, F., Di Felice, F. and Stern, F. "Measurements of the velocity field around the Delft 372 catamaran in steady drift," $5^{\text {th }}$ World Maritime Technology Conference, November 37, Rhode Island, USA, 2015.

Eça, L. and Hoekstra, M. "A procedure for the estimation of the numerical uncertainty of CFD calculations based on grid refinement studies," Journal of Computational Physics, Vol. 262, 2014, pp. 104-130. 
Egeberg T.F., Yoon, H., Stern, F., Pettersen, B. and Bhushan, S. "3D measurements of vortex shedding from a ship hull by means of tomographic PIV." Proceedings of the ASME 33rd International Conference on Ocean, Offshore \& Arctic Engineering OMAE2014, June 8-13, San Francisco, USA, 2014.

Egolf, T.A., Wake, B. and Berezin, C. "Recent Rotor Wake Simulation and Modeling Studies at United Technologies Corporation," Proceedings 38th Aerospace Sciences Meeting and Exhibit, no. 2000-0115, 2000

Greenwell, DI. "Engineering models of delta wing vortex breakdown and its Effect on aerodynamic characteristics." Chapter 21, RTOTR-AVT-080, 2009.

Gorski, J.J., Miller, R.W. and Coleman, R.M. "The Use of a RANS Code in the Design and Analysis of a Naval Combatant." $25^{\text {th }}$ Symposium on Naval Hydrodynamics, St. John's, Newfoundland and Labrador, CANADA, 8-13 August 2004.

Gorski, J.J., Haussling, H.J., Percival, A.S, Shaughnessy, J.J. and Buley, G.M. "An Investigation of Propeller Inflow for Naval Surface Combatants." 24th Symposium on Naval Hydrodynamics Fukuoka, JAPAN, 8-13 July 2002.

Gursul, I. "Unsteady flow phenomena over delta wings at high angle of attack." AIAA J., Vol. 32, No. 2, 1994, pp. 225-231.

Gursul, I. "Unsteady aspects of leading-edge vortices," Chapter 6, RTO-TR-AVT-080, 2009.

Huang, J., Carrica, P.M. and Stern, F. "Semi-Coupled Air/Water Immersed Boundary Approach for Curvilinear Dynamic Overset Grids with Application to Ship Hydrodynamics," International Journal Numerical Methods Fluids, Vol. 58, No. 6, 2008, pp. 591624.

ITTC 2002. "Testing and Extrapolation Methods Resistance Uncertainty Analysis, Example for Resistance Test," $23^{\text {rd }}$ ITTC International Towing Tank Conference, 2002.

Kim, S.E., Rhee, B.J. and Miller, R.W. "Anatomy of Turbulent Flow Around DARPA SUBOFF Body in a Turning Maneuver Using High-fidelity RANS Computations," International Shipbuilding Progress, Vol. 60, No. 1, 2014, pp. 207-231.

Larsson, L., Stern, F. and Visonneau, M. "Numerical Ship Hydrodynamics: An Assessment of the Gothenburg 2010 Workshop,” Springer, 2014, ISBN: 978-94-007-7188-8, 318 pages.

Longo, J. and Stern, F. "Uncertainty Assessment for Towing Tank Tests with Example for Surface Combatant DTMB Model 5415," Journal Ship Research, Vol. 49, No. 1, 2005, pp. 55-68.

Longo, J., Shao, J., Irvine, M., and Stern, F., "Phase-Averaged PIV for the Nominal Wake of a Surface Ship in Regular Head Waves," ASME Journal of Fluids Engineering, Vol. 129, 2007, pp. 524-540.

Menter, F.R. "Two-equation Eddy-viscosity Turbulence Models for Engineering Applications," AIAA Journal, Vol. 32, 1994, pp. 1598160.

Nelson, R.C., and Pelletier, A. "The unsteady aerodynamics of slender wings and aircraft undergoing large amplitude maneuvers." Progress in Aerospace Sciences, Vol. 39, 2003, pp. 185-248.

Olivieri, A., Pistani. F., Avanzini, A., Stern, F. and Penna, R. "Towing Tank Experiments of Resistance, Sinkage and Trim, Boundary Layer, Wake, and Free-surface Flow Around a Naval Combatant INSEAN 2340 Model," Iowa Institute of Hydraulic Research, The University of Iowa, IIHR Report No. 421, September 2001, 56 pp.

Simpson, RL. "Aspects of Turbulent Boundary Layer Separation," Prog Aerospace Sci, Vol. 32, No. 5, 1996, pp. 457-521.

Simpson, R. L., Walker, D.A. and Shinpaugh, K.A. "Description of a 1000 Sensor Constant Current Anemometer System for Locating Three-Dimensional Turbulent Boundary Layer Separations," Report VPI-AOE-185, distributed by DTIC for Defense Advanced Research Projects Agency, 1992.
Spalart, P. "Detached eddy simulation." Annual Rev. Fluid Mech. 41, 2009, pp. 181-202

Stern, F., Wilson, R.V., Coleman, H.W. and Paterson, E.G., "Comprehensive Approach to Verification and Validation of CFD Simulations-Part 1: Methodology and Procedures," Journal of Fluids Engineering - Transactions of ASME, Vol. 123, 2001, pp. 793-802.

Stern, F., Agdrup, K., Kim, S.Y., Hochbaum, A.C., Rhee, K.P., Quadvlieg, F., Perdon, P., Hino, T., Broglia, R. and Gorski, J. "Experience from SIMMAN 2008: The First Workshop on Verification and Validation of Ship Maneuvering Simulation Methods," Journal of Ship Research, Vol. 55, No. 2, 2011, pp. 135-147.

Surana, A., Grunberg, O. and Haller. G. "Exact Theory of Threedimensional Flow Separation. Part I: Steady Separation," J. Fluid Mech., Vol. 564, 2006, pp. 57-103.

Toxopeus, S.L., Atsavapranee, P., Wolf, E., Daum, S., Pattenden, R.J., Widjaja, R., Zhang, J.T. and Gerber, A.G. "Collaborative CFD Exercise for a Submarine in a Steady Turn," $31^{\text {st }}$ International Conference on Ocean, Offshore and Arctic Engineering (OMAE), Rio de Janeiro, Brazil, July, 2012.

Vaz, G.; Jaouen, F. A. P. and Hoekstra, M. "Free-Surface Viscous Flow Computations. Validation of URANS Code FRESCO," 28th International Conference on Ocean, Offshore and Arctic Engineering (OMAE), Honolulu, Hawaii, May 31-June 5, 2009.

Wackers, J., Deng, G.B., Leroyer, A., Queutey, P., Visonneau, M. "Adaptive Grid Refinement Algorithm for Hydrodynamic Flows," Computers and Fluids, Vol. 55, 2012, pp.80-100.

Xing, T., Kandasamy, M. and Stern, F., "Unsteady Free-Surface WaveInduced Separation: Analysis of Turbulent Structures using Detached Eddy Simulation and Single-Phase Level Set," Journal of Turbulence, Vol. 8, No. 44, 2007, pp. 1-35.

Xing, T. and Stern, F. "Factors of safety for Richardson extrapolation," Journal of Fluids Engineering-Transactions of ASME, Vol. 132, 2010, 061403.

Xing, T., Bhushan, S. and Stern F. "Unsteady Vortical Flow and Turbulent Structures for a Tanker Hull Form at Large Drift Angles," Ocean Engineering, Vol. 55, 2012, pp. 23-43.

Yoon, H., Gui, L., Bhushan, S., and Stern, F., "Tomographic PIV Measurements for a Surface Combatant at Straight Ahead and Static Drift Conditions," 30th Symposium on Naval Hydrodynamics, Hobart, Tasmania, Australia, 2014.

Zhang Z.J. and Stern, F. "Free-surface Wave-induced Separation," J. Fluids. Eng. Vol. 118, 1996, pp. 546-554. 
Table 1: Summary of CFD solvers, their numerical methods and models, and submitted results for the straight-ahead $\beta=0$ and static drift $\beta=20$ degree conditions.

\begin{tabular}{|c|c|c|c|c|c|c|c|c|}
\hline \multirow{2}{*}{$\begin{array}{l}\text { Organization/ } \\
\text { code name }\end{array}$} & \multirow{2}{*}{$\begin{array}{l}\text { Discretization methods } \\
\text { and schemes }\end{array}$} & \multirow{2}{*}{ Case } & \multicolumn{3}{|c|}{ Modeling } & \multicolumn{2}{|c|}{ Grid characteristics } & \multirow{2}{*}{ Results submitted } \\
\hline & & & Turbulence & Wall boundary & Free-surface & Type/Size & Resolution & \\
\hline \multirow{2}{*}{$\begin{array}{l}\text { IIHR/ } \\
\text { CFDShip-Iowa } \\
\text { v4.5 }\end{array}$} & \multirow{2}{*}{$\begin{array}{l}\text { Finite difference, Hybrid } \\
2^{\text {nd }} / 4^{\text {th }} \text { order upwind for } \\
\text { convection, } 2^{\text {nd }} \text { order } \\
\text { centered for diffusion; } \\
\text { PISO/ Projection for P-V } \\
\text { coupling }\end{array}$} & $\beta=0 \mathrm{deg}$ & \multirow{2}{*}{$\begin{array}{l}\text { EARSM } \\
\text { DES }\end{array}$} & \multirow{2}{*}{$\begin{array}{l}\text { Low Re near wall } \\
\text { turbulence model, } \\
\text { no slip condition }\end{array}$} & \multirow[b]{2}{*}{ Level-set } & $\begin{array}{l}\text { Overset multiblock structured; } \\
10 \mathrm{M}, 50 \mathrm{M} \text { and } 84 \mathrm{M} \text { points }\end{array}$ & $\begin{array}{c}\text { Refined grid with } \Delta=0.00122 \\
L_{P P} \text { all along the hull; } 7-8 \text { grid } \\
\text { points in SDV core for finest } \\
\text { grid }\end{array}$ & $\begin{array}{l}\text { Forces, wave-elevation and profiles, } 3 \mathrm{D} \text { vortical } \\
\text { structures, vortex core analysis, planar and volume } \\
\text { plots, onset and separation analysis }\end{array}$ \\
\hline & & $\beta=20 \mathrm{deg}$ & & & & $\begin{array}{l}\text { Overset multiblock structured; } \\
10 \mathrm{M}, 50 \mathrm{M}, 84 \mathrm{M} \text { and } 250 \mathrm{M} \text { points }\end{array}$ & $\begin{array}{l}\text { Refined grid with } \Delta=0.00122 L_{P P} \\
\text { all along the primary vortices; } \\
14-15 \text { grid points in SDTV core } \\
\text { on finest grid }\end{array}$ & $\begin{array}{c}\text { Forces and Moments, wave-elevation and profiles, } \\
\text { 3D vortical structures, vortex core analysis, planar } \\
\text { and limited volume plots, onset and separation } \\
\text { analysis of mean and instantaneous vortices, } \\
\text { instability and TKE budget analysis }\end{array}$ \\
\hline \multirow{2}{*}{$\begin{array}{l}\text { ECN-CNRS/ } \\
\text { ISIS-CFD v3.1 }\end{array}$} & \multirow{2}{*}{$\begin{array}{l}\text { Finite Volume, } 2^{\text {nd }} \text { order } \\
\text { upwind for convection, } \\
2^{\text {nd }} \text { order centered for } \\
\text { diffusion; SIMPLE for P- } \\
\text { V coupling }\end{array}$} & $\beta=0 \mathrm{deg}$ & $\begin{array}{l}\mathrm{k}-\omega \mathrm{SST} \\
\text { EARSM } \\
\text { DES-SST }\end{array}$ & $\begin{array}{l}\text { Low Re near wall } \\
\text { turbulence model, } \\
\text { no slip condition }\end{array}$ & $\mathrm{VoF}$ & $\begin{array}{l}\text { Hexpress Hexahedra unstructured; } \\
\text { Coarse grid: } 22 \mathrm{M} \text { points, Fine } \\
\text { grid: } 81 \mathrm{M} \text { points }\end{array}$ & $\begin{array}{l}\text { Refined grid with } \Delta= \\
0.00033 \mathrm{~L}_{P P} \text { up to } \mathrm{x} / \mathrm{L}=0.6 ; 10 \\
\text { grid points in } \mathrm{SDV} \text { core for } \\
\text { finest grid }\end{array}$ & $\begin{array}{c}\text { Forces, wave-elevation and profiles, 3D vortical } \\
\text { structures, vortex core analysis, planar and volume } \\
\text { plots }\end{array}$ \\
\hline & & $\beta=20 \mathrm{deg}$ & $\begin{array}{l}\mathrm{k}-\omega \text { SST } \\
\text { EARSM } \\
\text { DES-SST }\end{array}$ & $\begin{array}{l}\text { Low Re near wall } \\
\text { turbulence model, } \\
\text { no slip condition }\end{array}$ & $\mathrm{VoF}$ & $\begin{array}{c}\text { Hexpress Hexahedra unstructured; } \\
\text { 27M points and Coarse grid + } \\
\text { AGR : } 10 \mathrm{M} \text { points }\end{array}$ & $\begin{array}{l}\text { Grid adaption limited to early } \\
\text { part of the vortex progression }\end{array}$ & $\begin{array}{l}\text { Forces and Moments, wave-elevation and profiles, } \\
\text { 3D vortical structures, planar plots, limited volume } \\
\text { plots }\end{array}$ \\
\hline \multirow{2}{*}{$\begin{array}{l}\text { MARIN/ } \\
\text { ReFRESCO } \\
\text { V2.0.0 }\end{array}$} & \multirow{2}{*}{$\begin{array}{l}\text { Finite Volume, } 2^{\text {nd }} \text { order } \\
\text { upwind for convection, } \\
2^{\text {nd }} \text { order centered for } \\
\text { diffusion; SIMPLE for P- } \\
\text { V coupling }\end{array}$} & $\beta=0 \mathrm{deg}$ & $\begin{array}{c}\mathrm{k}-\omega \mathrm{SST}(1994 \\
\text { and } 2003 \text { versions }) \\
\text { KSKL }\end{array}$ & $\begin{array}{c}\text { Low Re near wall } \\
\text { turbulence model, } \\
\text { no slip condition }\end{array}$ & $\begin{array}{l}\text { Double-body } \\
\text { and } \\
\text { VoF }\end{array}$ & $\begin{array}{l}\text { Hexpress Hexahedra } \\
\text { unstructured/GridPro Multiblock } \\
\text { structured; } 45 \mathrm{M} \text { points ( } 6 \text { coarser } \\
\text { grids up to } 703 \mathrm{~K} \text { points) }\end{array}$ & No vortex refinement & $\begin{array}{l}\text { Forces, wave-elevation and profiles, 3D vortical } \\
\text { structures, vortex core analysis, planar and volume } \\
\text { plots }\end{array}$ \\
\hline & & $\beta=20 \mathrm{deg}$ & $\begin{array}{c}\mathrm{k}-\omega \mathrm{SST}(1994 \\
\text { and } 2003 \text { versions })\end{array}$ & $\begin{array}{c}\text { Low Re near wall } \\
\text { turbulence model, } \\
\text { no slip condition }\end{array}$ & Double-body & $\begin{array}{l}\text { Hexpress Hexahedra unstructured/ } \\
\text { GridPro Multiblock structured; } \\
33.6 \mathrm{M} \text { points ( } 7 \text { coarser grids up } \\
\text { to } 1.3 \mathrm{M} \text { points })\end{array}$ & $\begin{array}{l}\text { Intermediate grid refinement } \\
\text { among computations }\end{array}$ & $\begin{array}{c}\text { Forces and Moments, 3D vortical structures, } \\
\text { planar and volume plots }\end{array}$ \\
\hline \multirow{2}{*}{$\begin{array}{l}\text { NSWCCD/ } \\
\text { NavyFOAM }\end{array}$} & \multirow{2}{*}{$\begin{array}{l}\text { Finite Volume, } 2^{\text {nd }} \text { order } \\
\text { upwind for convection, } \\
2^{\text {nd }} \text { order centered for } \\
\text { diffusion; PIMPLE for P- } \\
\text { V coupling }\end{array}$} & $\beta=0 \mathrm{deg}$ & $\mathrm{k}-\omega(2003)$ & $\begin{array}{l}\text { Wall function } \\
\text { condition } \\
\mathrm{y}^{+}=60\end{array}$ & VoF & $\begin{array}{c}\text { BoxerMesh Hexahedra } \\
\text { unstructured; } 9 \mathrm{M}, 24 \mathrm{M}, 51 \mathrm{M} \\
\text { points for half domain } \\
\text { (18M, 48M and 102M for full- } \\
\text { domain) }\end{array}$ & $\begin{array}{l}\text { Refined grid all along the hull; } \\
\text { Grid resolution similar to } \\
\text { CFDShip-Iowa and ISIS-CFD }\end{array}$ & $\begin{array}{l}\text { Forces, wave-elevation and profiles, 3D vortical } \\
\text { structures, planar plots, and limited volume plots }\end{array}$ \\
\hline & & $\beta=20 \mathrm{deg}$ & $\mathrm{k}-\omega(2003)$ & $\begin{array}{l}\text { Wall function } \\
\text { condition } \\
\mathrm{y}^{+}=60\end{array}$ & VoF & $\begin{array}{l}\text { BoxerMesh Hexahedra } \\
\text { unstructured; } 18 \mathrm{M}, 20 \mathrm{M} \text { and } 68 \mathrm{M} \\
\text { points; } 18 \mathrm{M} \text { and } 68 \mathrm{M} \text { grids } \\
\text { included side walls }\end{array}$ & $\begin{array}{l}\text { Grid refined to capture the } \\
\text { progression of vortices; } \\
\text { Grid resolution similar to } \\
\text { CFDShip-Iowa }\end{array}$ & $\begin{array}{l}\text { Forces and Moments, , wave-elevation and } \\
\text { profiles, 3D vortical structures, planar plots, and } \\
\text { limited volume plots }\end{array}$ \\
\hline \multirow{2}{*}{$\begin{array}{l}\text { STAR-CCM+/ } \\
\text { NTUA }\end{array}$} & \multirow{3}{*}{$\begin{array}{l}\text { Finite Volume, } 2^{\text {nd }} \text { order } \\
\text { discretization schemes } \\
\text { for convection and } \\
\text { diffusion; SIMPLE for P- } \\
\text { V coupling }\end{array}$} & $\beta=0$ deg & $\mathrm{k}-\varepsilon$ & $\begin{array}{l}\text { Wall-function } \\
\mathrm{y}^{+} \sim 30-40\end{array}$ & $\mathrm{VoF}$ & $\begin{array}{c}\text { Hexahedra unstructured; } \\
2.5 \mathrm{M}, 3 \mathrm{M}, 3.5 \mathrm{M} \text { and } 4.4 \mathrm{M} \text { points }\end{array}$ & $\begin{array}{l}\text { Lowest modes (cell) count } \\
\text { among computations }\end{array}$ & Forces and planar plots \\
\hline & & $\beta=20 \operatorname{deg}$ & $\mathrm{k}-\varepsilon$ & $\begin{array}{l}\text { Wall-function } \\
\mathrm{y}^{+} \sim 30-40\end{array}$ & VoF & $\begin{array}{l}\text { Hexahedra unstructured; } \\
4.6 \mathrm{M}, 5.7 \mathrm{M} \text { and } 7 \mathrm{M} \text { points }\end{array}$ & $\begin{array}{c}\text { Lowest nodes (cell) count among } \\
\text { computations }\end{array}$ & Forces and Moments and planar plots \\
\hline $\begin{array}{l}\text { STAR-CCM+/ } \\
\text { FORCE }\end{array}$ & & $\beta=20 \mathrm{deg}$ & $\mathrm{k}-\omega \mathrm{SST}$ & $\begin{array}{l}\text { Wall-function } \\
\mathrm{y}^{+} \sim 15\end{array}$ & $\mathrm{VoF}$ & $\begin{array}{l}\text { Hexahedra unstructured; } \\
\text { 7M points }\end{array}$ & $\begin{array}{l}\text { Grid is finer than NTUA grid, } \\
\text { but coarser than other grids }\end{array}$ & Forces and Moments and planar plots \\
\hline
\end{tabular}


Table 2: Comparison of CFD force and vortex extent predictions with experimental data for straight-ahead $\beta=0$ condition.

\begin{tabular}{|c|c|c|c|c|c|c|c|c|c|c|}
\hline \multirow{2}{*}{\multicolumn{4}{|c|}{ Experiment Facility/CFD Code }} & \multicolumn{3}{|c|}{$X(\mathrm{~N})$} & \multicolumn{4}{|c|}{ Extent of Vortices } \\
\hline & & & & \multirow{2}{*}{\begin{tabular}{|c|}
$\times 10^{-3}$ \\
-15.69 \\
\end{tabular}} & \multirow{2}{*}{$\begin{array}{c}E \% \mathrm{D} \\
\mathrm{n} / \mathrm{a} \\
\end{array}$} & \multirow[t]{2}{*}{ Uncertainty } & \multirow{2}{*}{$\begin{array}{c}\mathrm{SDV} \\
\mathrm{n} / \mathrm{a}\end{array}$} & \multirow{2}{*}{$\begin{array}{c}\mathrm{E} \% \mathrm{D} \\
\mathrm{n} / \mathrm{a} \\
\end{array}$} & \multirow{2}{*}{$\begin{array}{c}\text { FBKV } \\
\mathrm{n} / \mathrm{a}\end{array}$} & \multirow{2}{*}{$\begin{array}{c}\mathrm{E} \% \mathrm{D} \\
\mathrm{n} / \mathrm{a}\end{array}$} \\
\hline \multirow{2}{*}{\multicolumn{3}{|c|}{$\begin{array}{l}\text { Experiment/ Barehull (Longo et al., 2007) (D) } \\
\text { Experiment }(D) \text { (Yoon et al., 2014) }\end{array}$}} & \multirow{2}{*}{ PMM } & & & & & & & \\
\hline & & & & -16.63 & $\mathrm{n} / \mathrm{a}$ & $U_{D}=3.6 \% D$ & 0.4 & $\mathrm{n} / \mathrm{a}$ & $>1.0$ & $\mathrm{n} / \mathrm{a}$ \\
\hline Code (Institution) & Blockage & Turbulence Model & Grid size & & & & & & & \\
\hline \multirow{6}{*}{$\begin{array}{l}\text { CFDShip-IOWA } \\
\text { (IIHR) }\end{array}$} & \multirow{3}{*}{ No } & \multirow{3}{*}{ URANS } & $10 \mathrm{M}$ & -17.37 & $-4.46 \%$ & \multirow{3}{*}{$U_{S N}=2.2 \% S$} & & & & \\
\hline & & & $50 \mathrm{M}$ & -16.16 & $2.77 \%$ & & & & & \\
\hline & & & $84 \mathrm{M}$ & -16.86 & $1.36 \%$ & & 0.3 & $25 \%$ & 0.45 & $>55 \%$ \\
\hline & \multirow{3}{*}{ No } & \multirow{3}{*}{ DES } & $10 \mathrm{M}$ & -17.16 & $-3.19 \%$ & \multirow{3}{*}{$\begin{array}{c}\mathrm{U}_{\mathrm{SN}}= \\
1.25 \% S\end{array}$} & & & & \\
\hline & & & $50 \mathrm{M}$ & -16.49 & $0.84 \%$ & & & & & \\
\hline & & & $84 \mathrm{M}$ & -16.34 & $1.74 \%$ & & 0.53 & $-32.5 \%$ & $>1.0$ & $\mathrm{n} / \mathrm{a}$ \\
\hline \multirow{4}{*}{$\begin{array}{l}\text { ReFRESCO FS, } \\
\text { Barehull (MARIN) }\end{array}$} & \multirow{4}{*}{ Yes } & \multirow{4}{*}{ RANS } & $1.28 \mathrm{M}$ & -16.74 & $6.69 \%{ }^{\mathrm{BH}}$ & \multirow{4}{*}{$U_{S N}=1.7 \% S$} & & & & \\
\hline & & & $2.88 \mathrm{M}$ & -16.05 & $2.29 \%^{\mathrm{BH}}$ & & & & & \\
\hline & & & $10 \mathrm{M}$ & -15.76 & $0.45 \%{ }^{\mathrm{BH}}$ & & & & & \\
\hline & & & $45 \mathrm{M}$ & -15.68 & $0.06 \%{ }^{\mathrm{BH}}$ & & 0.6 & $-50 \%$ & 0.4 & $>60 \%$ \\
\hline \multirow{3}{*}{$\begin{array}{l}\text { ISIS-CFD } \\
\text { (ECN/CNRC) }\end{array}$} & \multirow{3}{*}{ No } & \multirow{2}{*}{ URANS } & $22 \mathrm{M}$ & -15.99 & $-3.83 \%$ & \multirow{3}{*}{-} & & & & \\
\hline & & & $81 \mathrm{M}$ & -15.6 & $-6.2 \%$ & & 0.7 & $-75 \%$ & 0.4 & $>60 \%$ \\
\hline & & DES & $81 \mathrm{M}$ & -14.46 & $-13.02 \%$ & & & & & \\
\hline \multirow{3}{*}{$\begin{array}{l}\text { NavyFOAM } \\
\text { (NSWCCD) }\end{array}$} & \multirow{3}{*}{ No } & \multirow{3}{*}{ URANS } & $9 \mathrm{M}$ & -16.27 & $-2.2 \%$ & \multirow{3}{*}{-} & & & & \\
\hline & & & $25 \mathrm{M}$ & -16.11 & $-3.2 \%$ & & & & & \\
\hline & & & $51 \mathrm{M}$ & -15.68 & $-5.7 \%$ & & $\mathrm{n} / \mathrm{a}$ & - & $\mathrm{n} / \mathrm{a}$ & - \\
\hline & & & $2.5 \mathrm{M}$ & -17.16 & $3.2 \%$ & & & & & \\
\hline STAR-CCM++ & No & RANS & $3 \mathrm{M}$ & -16.8 & $1.0 \%$ & & $\mathrm{n} / \mathrm{a}$ & - & $\mathrm{n} / \mathrm{a}$ & - \\
\hline (NTUA) & 100 & Кลบ & $3.5 \mathrm{M}$ & -16.7 & $0.4 \%$ & 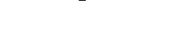 & & & & \\
\hline & & & $4.4 \mathrm{M}$ & -16.73 & $0.5 \%$ & & & & & \\
\hline Average $(\bar{E} \pm \mathrm{SD}$ & (D) & & & $-16.1 \pm 1.7 \%$ & $3.04 \%$ & $U_{V}=4 \% D$ & & & & \\
\hline
\end{tabular}

Shaded rows are the best results from each institution

BH \% errors are computed using barehull EFD data.

Table 3 Comparison of CFD forces and moment predictions with experimental data for static drift $\beta=20$ condition.

\begin{tabular}{|c|c|c|c|c|c|c|c|c|c|c|}
\hline \multirow{2}{*}{\multicolumn{4}{|c|}{ Experiment/CFD }} & \multicolumn{2}{|c|}{$X(\mathrm{~N})$} & \multicolumn{2}{|c|}{$Y(\mathrm{~N})$} & \multicolumn{2}{|c|}{$N(\mathrm{~N}-\mathrm{m})$} & \multirow{3}{*}{$\begin{array}{l}\text { Uncertainty } \\
U_{D}=3.87 \% D\end{array}$} \\
\hline & & & & \multirow{2}{*}{$\begin{array}{c}\times 10^{-3} \\
-28.57\end{array}$} & \multirow{2}{*}{$\begin{array}{c}E \% D \\
-\end{array}$} & \multirow{2}{*}{$\begin{array}{c}\times 10^{-3} \\
153.57\end{array}$} & \multirow{2}{*}{$\begin{array}{c}E \% D \\
-\end{array}$} & \multirow{2}{*}{$\begin{array}{l}\times 10^{-3} \\
59.86\end{array}$} & \multirow{2}{*}{$\begin{array}{c}E \% D \\
-\end{array}$} & \\
\hline \multicolumn{3}{|c|}{ Experiment (Yoon et al., 2014) $D$} & PMM & & & & & & & \\
\hline Code (Institution) & Blockage & $\begin{array}{l}\text { Turbulence } \\
\text { Model }\end{array}$ & Grid size & & & & & & & \\
\hline \multirow{7}{*}{ CFDShip-IOWA (IIHR) } & \multirow{3}{*}{ No } & \multirow{3}{*}{ URANS } & $10 \mathrm{M}$ & -29.54 & 3.39 & 167.04 & 8.77 & 61.51 & 2.75 & \multirow{3}{*}{$U_{S N}=2.2 \% S$} \\
\hline & & & $48 \mathrm{M}$ & -30.03 & 5.1 & 159.54 & 3.9 & 60.21 & 0.6 & \\
\hline & & & $84 \mathrm{M}$ & -30.02 & 5.1 & 159.42 & 3.8 & 60.20 & 0.6 & \\
\hline & \multirow{4}{*}{ No } & \multirow{4}{*}{ DES } & $10 \mathrm{M}$ & -28.79 & 0.8 & 160.88 & 4.8 & 60.42 & 0.9 & \multirow{4}{*}{$U_{S N}=1.25 \% S$} \\
\hline & & & $48 \mathrm{M}$ & -29.79 & 4.3 & 158.21 & 3.0 & 59.97 & 0.2 & \\
\hline & & & $84 \mathrm{M}$ & -29.84 & 4.5 & 158.36 & 3.1 & 60.01 & 0.25 & \\
\hline & & & $250 \mathrm{M}$ & -29.96 & 4.9 & 158.60 & 3.3 & 58.55 & -2.2 & \\
\hline STAR-CCM+ (FORCE) & No & RANS & $7 \mathrm{M}$ & -32.3 & 13.1 & 160.7 & 4.6 & 63.1 & 5.4 & - \\
\hline ReFRESCO (MARIN) - DB & Yes & RANS & $34 \mathrm{M}$ & -23.81 & -20.2 & 127.70 & -16.6 & 55.42 & -7.4 & $U_{S N}=1.7 \% \mathrm{~S}$ \\
\hline \multirow{3}{*}{ ISIS-CFD (ECN/CNRC) } & \multirow{3}{*}{ No } & \multirow{2}{*}{ URANS } & $10 \mathrm{M}-\mathrm{AGR}$ & -28.47 & -0.35 & 159.95 & 4.16 & 59.97 & 0.18 & \multirow{3}{*}{ - } \\
\hline & & & $27 \mathrm{M}$ & -30.05 & 5.17 & 154.98 & 0.92 & 58.71 & -1.93 & \\
\hline & & DES & $27 \mathrm{M}$ & -28.06 & -1.78 & 153.24 & -0.22 & 60.88 & 1.70 & \\
\hline \multirow{3}{*}{ NavyFOAM (NSWCCD) } & No & \multirow{3}{*}{ URANS } & $18 \mathrm{M}$ & -26.5 & -7.2 & 166.1 & 8.2 & 52.4 & -12.4 & \multirow{3}{*}{ - } \\
\hline & Yes & & $20 \mathrm{M}$ & -26.5 & -7.2 & 175.4 & 14.2 & 53.4 & -10.9 & \\
\hline & Yes & & $68 \mathrm{M}$ & -27.27 & -4.5 & 163.6 & 6.5 & 55.66 & -7.0 & \\
\hline \multirow{2}{*}{ STAR-CCM+ (NTUA) } & \multirow{2}{*}{ No } & \multirow{2}{*}{ RANS } & $4.6 \mathrm{M}$ & -28.43 & -0.6 & 161.5 & 4.1 & 57.3 & -4.3 & \multirow{3}{*}{ - } \\
\hline & & & $5.7 \mathrm{M}$ & -28.17 & -1.4 & 154.09 & 0.3 & 57.44 & -4.0 & \\
\hline STAR-CCM+ (FORCE) & No & RANS & $7.0 \mathrm{M}$ & -28.3 & -0.9 & 154.62 & 0.7 & 57.23 & -4.4 & \\
\hline \multicolumn{3}{|l|}{ Average $(\bar{E} \pm$ SD $\% D)$} & & $-29.2 \pm 3 \%$ & $2.2 \%$ & $159.3 \pm 1 \%$ & $3.7 \%$ & $59.2 \pm 2.1 \%$ & $1.04 \%$ & $U_{V}=4.23 \% D$ \\
\hline
\end{tabular}

Shaded rows are the best results from each institution 
Table 4: Summary of vortices observed in the experimental data and CFDShip-Iowa URANS and DES, including onset, separation and pregression characteristics for straight-ahead $\beta=0$ and static drift $\beta=20$ degree conditions.

\begin{tabular}{|c|c|c|c|c|}
\hline Case & Side & Vortex & Label & Onset, separation and progression \\
\hline \multirow{7}{*}{$\beta=0 \mathrm{deg}$} & \multirow{7}{*}{$\begin{array}{l}\text { Port and } \\
\text { Stern }\end{array}$} & Sonar dome & SDV & $\begin{array}{l}\text { - Extends up to } x / L_{P P}=0.4 \text { in experiment, } 0.4 \text { and } 0.3 \text { in CFDShip-Iowa DES and URANS, respectively } \\
\text { - Onset on sonar dome surface, } \mathrm{X}=0.065, \mathrm{Z}=-0.055 \text {; Open-type separation without BL separation } \\
\text { - Advected by streamwise velocity away from the sonar dome }\end{array}$ \\
\hline & & $\begin{array}{l}\text { Sonar dome } \\
\text { trailing edge }\end{array}$ & SDTEV & $\begin{array}{l}\text { - Extends up to } x / L_{P P} \sim 0.12 \text { and } 0.2 \text { in experiments and DES, respectively. Not predicted in URANS. } \\
\text { - Onset on convex section of the sonar dome keel, } \mathrm{X}=0.109, \mathrm{Z}=-0.054 \text {; Open-type separation }\end{array}$ \\
\hline & & Fore-body keel & FBKV & $\begin{array}{l}\text { - Extends up to } x / L_{P P}>1.0 \text { in both data and CFDShip-Iowa DES, and up to } x / L_{P P}=0.45 \text { in CFDShip-Iowa URANS } \\
\text { - Onset from concave section of the sonar dome close to the keel, } \mathrm{X}=0.107, \mathrm{Z}=-0.0461 \\
\text { - Open-type separation with boundary layer separation at the sonar dome-keel intersection }\end{array}$ \\
\hline & & Bilge keel & BKV & - Onset from the bilge keel (BK) leading edge at $\mathrm{x}=0.6$; Open-type separation \\
\hline & & After-body keel & $\mathrm{ABKV}$ & $\begin{array}{l}\text { - Onset from the after body keel } \\
\text { - Open-type separation; vortex is advected by the streamwise velocity and moves closer to the hull }\end{array}$ \\
\hline & & Mid-hull & MHV & $\begin{array}{l}\text { - Predicted only by CFDShip-Iowa DES on } 84 \mathrm{M} \text { grid. Vortex extends up to } x / L_{P P}>1.0 \\
\text { - Sepration from the hull surface, } x / L_{P P}=0.5, y / L_{P P}=0.022, z / L_{P P}=-0.045 ; \text { Open-type separation } \\
\text { - Advected by the axial velocity upwards from the keel and away from the hull }\end{array}$ \\
\hline & & Transom vortices & & $\begin{array}{l}\text { - Karman-type transom vortex shedding. DES also predicts superimposed small scale turbulence structures } \\
\text { - Closed separation from the transom edge; Vortices are shed from the transom and advected in the transom wake }\end{array}$ \\
\hline \multirow{11}{*}{$\begin{array}{l}\beta=20 \\
\operatorname{deg}\end{array}$} & \multirow{8}{*}{ Leeward } & Sonar dome tip & SDTV & $\begin{array}{l}\text { - Clockwise rotating vortex; } \\
\text { - Formed due to merger of open-type separations; Shows helical mode instability }\end{array}$ \\
\hline & & $\begin{array}{c}\text { Sonar dome } \\
\text { separation bubble }\end{array}$ & SDSB & $\begin{array}{l}\text { - Closed separation } \\
\text { - Originates from sink node at the leading edge, encased by saddle point, and terminates at focii }\end{array}$ \\
\hline & & $\begin{array}{c}\text { Sonar dome } \\
\text { bubble vortex }\end{array}$ & LW-SDBV & $\begin{array}{l}\text { - Counter-rotating with respect to SDTV } \\
\text { - Onset from saddle point at } \mathrm{x} / \mathrm{L}_{\mathrm{PP}}=0.067, \mathrm{z} / \mathrm{L}_{\mathrm{PP}}=-0.0465 \text { on hull; Open-closed separation }\end{array}$ \\
\hline & & $\begin{array}{l}\text { free-surface } \\
\text { vortex }\end{array}$ & LW-FSV & $\begin{array}{l}\text { - Counter-rotating with respect to SDTV } \\
\text { - Onset from saddle point at } \mathrm{x} / \mathrm{L}_{\mathrm{PP}}=0.083, \mathrm{z} / \mathrm{L}_{\mathrm{PP}}=-0.015 \text {; Open-closed type separation }\end{array}$ \\
\hline & & Fore-body keel & LW-FBKV & $\begin{array}{l}\text { - Counter-rotating with respect to SDTV } \\
\text { - Vortex onset from the hull surface at } \mathrm{x} / \mathrm{L}_{\mathrm{PP}}=0.19, \mathrm{z} / \mathrm{L}_{\mathrm{PP}}=-0.037 \text {; Open-type separation }\end{array}$ \\
\hline & & Bilge keel vortex & BKV & $\begin{array}{l}\text { - Primary clockwise rotating vortex from BK tip, and counter-rotating vortex from the leeward side of BK } \\
\text { - Both primary and secondary separations are open-type. }\end{array}$ \\
\hline & & Mid hull vortex & LW-MHV & - Co-rotating with SDTV; Onset at $\mathrm{x} / \mathrm{L}_{\mathrm{PP}}=0.655, \mathrm{z} / \mathrm{L}_{\mathrm{PP}}=-0.039 ;$ Open-type separation \\
\hline & & After-body keel & $\mathrm{ABKV}$ & - Co-rotating with respect to SDTV; Onset from after body keel; Open-type separation \\
\hline & \multirow{3}{*}{ Windward } & $\begin{array}{l}\text { Free-surface } \\
\text { vortex }\end{array}$ & WW-FSV & $\begin{array}{l}\text { - Closed separation } \\
\text { - Separates from source node at bow } \mathrm{x} / \mathrm{L}_{\mathrm{PP}}=-0.0035, \mathrm{z} / \mathrm{L}_{\mathrm{PP}}=0.004 \text {, and attached at saddle point on free-surface }\end{array}$ \\
\hline & & Bilge keel tip & BKTV & $\begin{array}{l}\text { - Primary vortex from bilge keel edge, and secondary vortices from the windward side of the bilge keel } \\
\text { - Primary vortex is co-rotating with respect to SDTV } \\
\text { - Primary vortex has open-type separation from bilge keel leading edge; Primary BKTV has helical mode instability }\end{array}$ \\
\hline & & Fore-body keel & $\begin{array}{l}\text { WW- } \\
\text { FBKV }\end{array}$ & $\begin{array}{l}\text { - Co-rotating with respect to SDTV } \\
\text { - Open-type separation from leeward side hull at } \mathrm{x} / \mathrm{L}_{\mathrm{PP}}=0.34, \mathrm{z} / \mathrm{L}_{\mathrm{PP}}=-0.043\end{array}$ \\
\hline
\end{tabular}

Table 5: Summary of axial vortex core prediction errors for straight-ahead $\beta=0$ and static drift $\beta=20$ conditions.

\begin{tabular}{|c|c|c|c|c|c|c|c|c|c|c|c|c|}
\hline \multirow[b]{2}{*}{ Case } & \multirow{2}{*}{ Vortex } & \multirow{2}{*}{ CFD } & \multicolumn{5}{|c|}{ Flow variable prediction error, $\% \mathrm{D}$} & \multirow{2}{*}{$\begin{array}{l}\text { Solver } \\
\bar{E} \% D\end{array}$} & \multirow{2}{*}{$\begin{array}{c}\text { Overall } \\
\bar{E} \pm \mathrm{SD} \% D\end{array}$} & \multirow[b]{2}{*}{$\mathrm{U}_{\mathrm{SN}} \% \mathrm{~S}$} & \multirow{2}{*}{$\mathrm{U}_{\mathrm{D}} \% D$} & \multirow{2}{*}{$\mathrm{U}_{\mathrm{V}} \% D$} \\
\hline & & & Location & $\omega_{\mathrm{x}}$ & $\mathrm{Q}$ & $\mathrm{U}_{\mathrm{x}}$ & TKE & & & & & \\
\hline \multirow{8}{*}{$\beta=0 \mathrm{deg}$} & \multirow{4}{*}{ SDV } & CFDShip-Iowa-URANS & 12.3 & 14.7 & 44.5 & 10.1 & 45.9 & 25.5 & \multirow{4}{*}{$72.9 \pm 42 \%$} & 9.9 & \multirow{4}{*}{8.6} & 13.1 \\
\hline & & CFDShip-Iowa-DES & 7.0 & 130.7 & 197.2 & 16.2 & 72.8 & 84.8 & & 11.1 & & 14.0 \\
\hline & & ISIS-CFD -URANS & 16 & 184 & 315 & 11.46 & 23.6 & 110.0 & & - & & - \\
\hline & & ReFRESCO - RANS & 19 & 133.4 & 183 & 11.5 & 27 & 71.4 & & - & & - \\
\hline & \multirow{4}{*}{ FBKV } & CFDShip-Iowa-URANS & 12.0 & 22.2 & 44.7 & 5.1 & 44.2 & 25.6 & \multirow{4}{*}{$53.1 \pm 63 \%$} & 14.6 & \multirow{4}{*}{7.3} & 16.3 \\
\hline & & CFDShip-Iowa-DES & 5.1 & 154 & 297 & 1.93 & 90.9 & 109.8 & & 17.9 & & 19.7 \\
\hline & & ISIS-CFD -URANS & 12.3 & 35.2 & 61.7 & 3.77 & 57.3 & 34.1 & & - & & - \\
\hline & & ReFRESCO - RANS & 26.4 & 88.8 & 54.5 & 7.2 & 37.2 & 42.8 & & - & & - \\
\hline \multirow{7}{*}{$\begin{array}{l}\beta=20 \\
\text { deg }\end{array}$} & \multirow{4}{*}{ SDTV } & CFDShip-Iowa-URANS & 3.8 & 54.6 & 48.7 & 34.7 & 86.2 & 45.6 & \multirow{4}{*}{$49.4 \pm 24 \%$} & 26.7 & \multirow{4}{*}{9.1} & 28.2 \\
\hline & & \begin{tabular}{|l|} 
CFDShip-Iowa-DES \\
\end{tabular} & 1.22 & 20.2 & 37.4 & 35.9 & 67.5 & 32.4 & & 13.8 & & 16.5 \\
\hline & & ISIS-CFD -URANS & 5.67 & 66.6 & 90.8 & 25.9 & 88.5 & 55.5 & & - & & - \\
\hline & & ReFRESCO - RANS & 11.5 & 69.6 & 84 & 57.4 & 97.3 & 64.0 & & & & - \\
\hline & \multirow{3}{*}{ BKTV } & CFDShip-Iowa-URANS & 6.1 & 28.5 & 148.6 & 2.9 & 48 & 46.8 & \multirow{3}{*}{$36.1 \pm 23 \%$} & 8.0 & \multirow{3}{*}{4.4} & 9.13 \\
\hline & & \begin{tabular}{|l|} 
CFDShip-Iowa-DES \\
\end{tabular} & 3.9 & 41.6 & 108.5 & 7.7 & 15.3 & 35.4 & & 22.9 & & 23.3 \\
\hline & & ISIS-CFD -URANS & 4.7 & 25.8 & 27.4 & 3.7 & 68.7 & 26.1 & & - & & - \\
\hline
\end{tabular}


Table 6: Summary of cross-plane vortex core prediction errors for straight-ahead $\beta=0$ and static drift $\beta=20$ conditions.

\begin{tabular}{|c|c|c|c|c|c|c|c|c|c|c|c|}
\hline \multirow{2}{*}{ Case } & \multirow{2}{*}{ Vortex } & \multirow{2}{*}{$\begin{array}{l}\text { Vortex } \\
\text { Location }\end{array}$} & \multirow{2}{*}{ CFD } & \multicolumn{5}{|c|}{ Flow variable, $\bar{E} \% D$} & \multirow{2}{*}{$\mathrm{U}_{\mathrm{SN}} \% S$} & \multirow{2}{*}{$\mathrm{U}_{\mathrm{D}} \% D$} & \multirow{2}{*}{$\mathrm{U}_{\mathrm{V}} \% D$} \\
\hline & & & & $\omega_{\mathrm{x}}$ & $\mathrm{Q}$ & $\mathrm{U}_{\mathrm{x}}$ & TKE & Overall & & & \\
\hline \multirow{4}{*}{$\begin{array}{l}\beta=0 \\
\text { deg }\end{array}$} & \multirow{2}{*}{ SDV } & \multirow{2}{*}{$\begin{array}{l}\text { Avg. } \mathrm{x} / \mathrm{L}=0.2 \\
\text { and } 0.3\end{array}$} & CFDShip-Iowa-URANS & 42.79 & 40.9 & 3.4 & 49.8 & 34.2 & 9.9 & \multirow{2}{*}{8.6} & 13.1 \\
\hline & & & CFDShip-Iowa-DES & 63.09 & 28.25 & 6.9 & 77.3 & 43.9 & 11.1 & & 14.0 \\
\hline & \multirow{2}{*}{ FBKV } & \multirow{2}{*}{$\begin{array}{c}\text { Avg. } \mathrm{x} / \mathrm{L}=0.2 \\
\text { and } 0.3\end{array}$} & CFDShip-Iowa-URANS & 41.65 & 73.05 & 12.1 & 58.7 & 46.4 & 14.6 & \multirow{2}{*}{7.3} & 16.3 \\
\hline & & & CFDShip-Iowa-DES & 55.5 & 91.6 & 12.85 & 65.15 & 56.3 & 17.9 & & 19.7 \\
\hline \multirow{4}{*}{$\begin{array}{l}\beta=20 \\
\operatorname{deg}\end{array}$} & \multirow{2}{*}{ SDTV } & \multirow{2}{*}{$\begin{array}{c}\text { Avg. } \mathrm{x} / \mathrm{L}=0.2, \\
0.4 \text { and } 0.8 \\
\end{array}$} & CFDShip-Iowa-URANS & 19.9 & 41 & 58.2 & 95.9 & 53.8 & 18.2 & \multirow{2}{*}{9.1} & 28.2 \\
\hline & & & CFDShip-Iowa-DES & 17.3 & 41.6 & 19.2 & 63.7 & 35.5 & 13.8 & & 16.5 \\
\hline & \multirow{2}{*}{ BKTV } & \multirow{2}{*}{$\mathrm{x} / \mathrm{L}=0.6$} & CFDShip-Iowa-URANS & 4.1 & 73.2 & 12.7 & 62.2 & 38.1 & 8.0 & \multirow{2}{*}{4.4} & 9.13 \\
\hline & & & CFDShip-Iowa-DES & 15.6 & 78.7 & 60.5 & 66.9 & 55.4 & 22.9 & & 23.3 \\
\hline
\end{tabular}

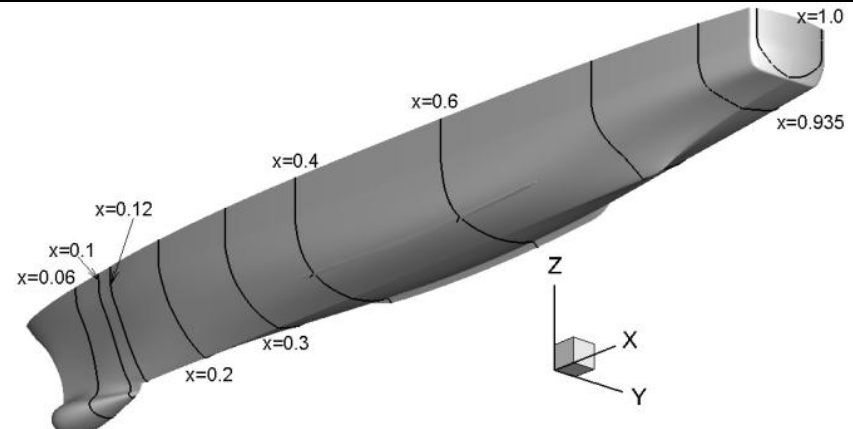

\begin{tabular}{|c|c|c|}
\hline & Full-scale & DTMB 5415 \\
\hline Scale & $1: 1$ & $1: 46.6$ \\
\hline $\mathrm{L}_{\mathrm{PP}}(\mathrm{m})$ & 142.0 & 3.048 \\
\hline $\mathrm{L}_{\mathrm{WL}}(\mathrm{m})$ & 142.18 & 3.052 \\
\hline $\mathrm{B}_{\mathrm{WL}}(\mathrm{m})$ & 19.10 & 0.410 \\
\hline $\mathrm{T}_{\mathrm{m}}(\mathrm{m})$ & 6.16 & 0.132 \\
\hline$\nabla\left(\mathrm{m}^{3}\right)$ & 8472.0 & 0.0086 \\
\hline $\mathrm{C}_{\mathrm{B}}$ & 0.506 & 0.506 \\
\hline
\end{tabular}

Figure 1: Coordinate system and measurement locations for surface combatant 5415 . The inset table shows the hull particulars.

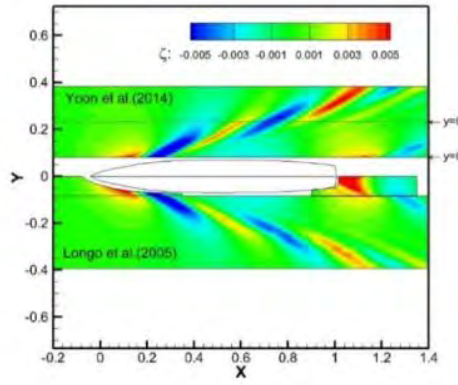

(a)

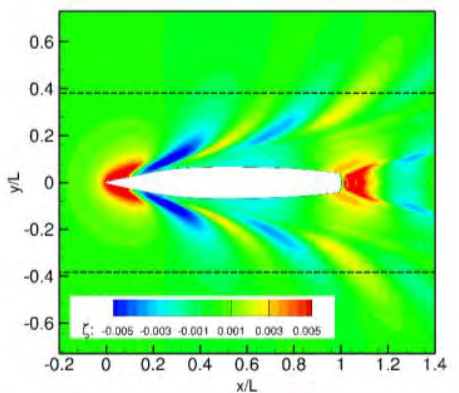

(d)

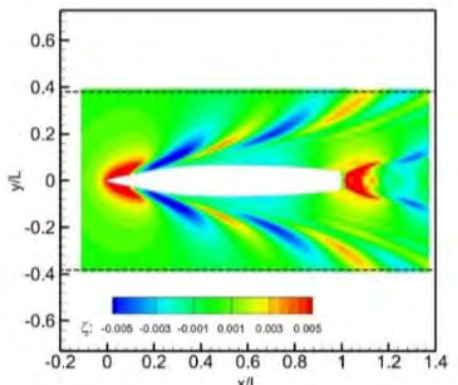

(b)

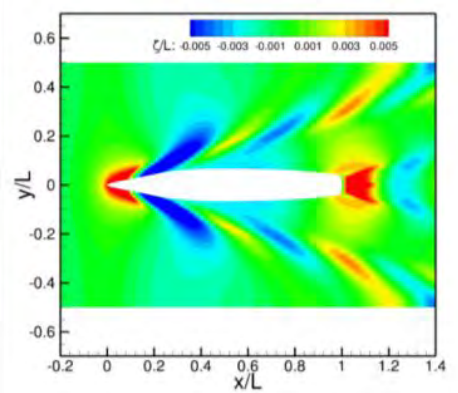

(e)

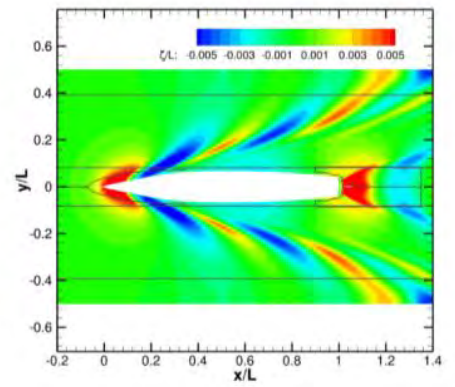

(C)

Figure 2: Wave contours obtained using: (a) experiment; (b) CFDShip-IOWA; (c) ReFRESCO; (d) ISIS-CFD; and (e) NavyFOAM for $\beta=0$.

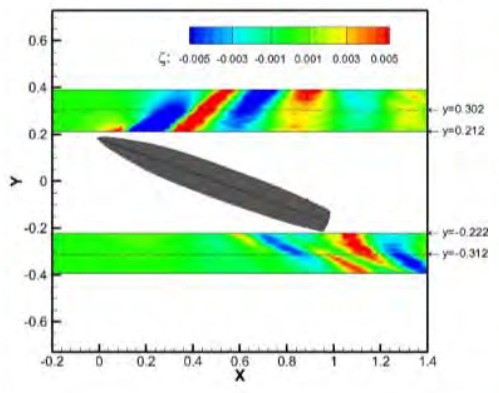

(a)

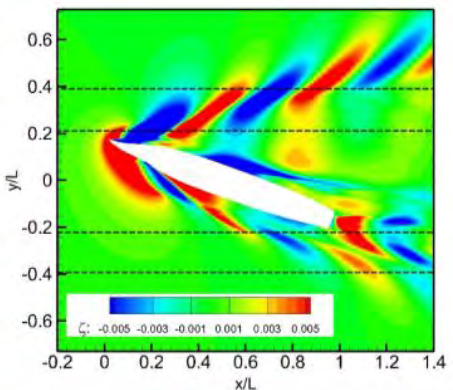

(b)

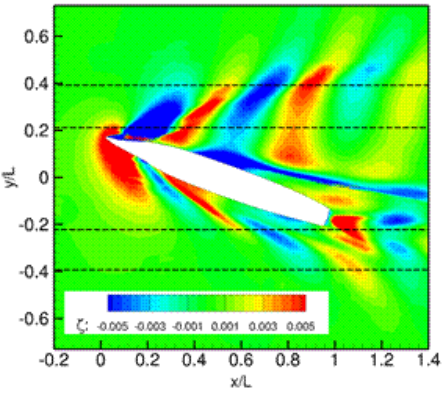

(c)

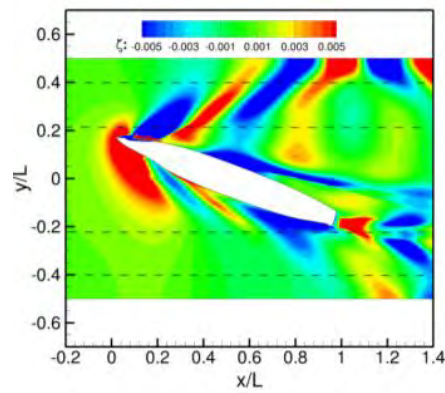

(d)

Figure 3: Wave contours obtained using: (a) experiment; (b) CFDShip-IOWA; (c) ISIS-CFD; and (d) NavyFOAM for $\beta=20$. 

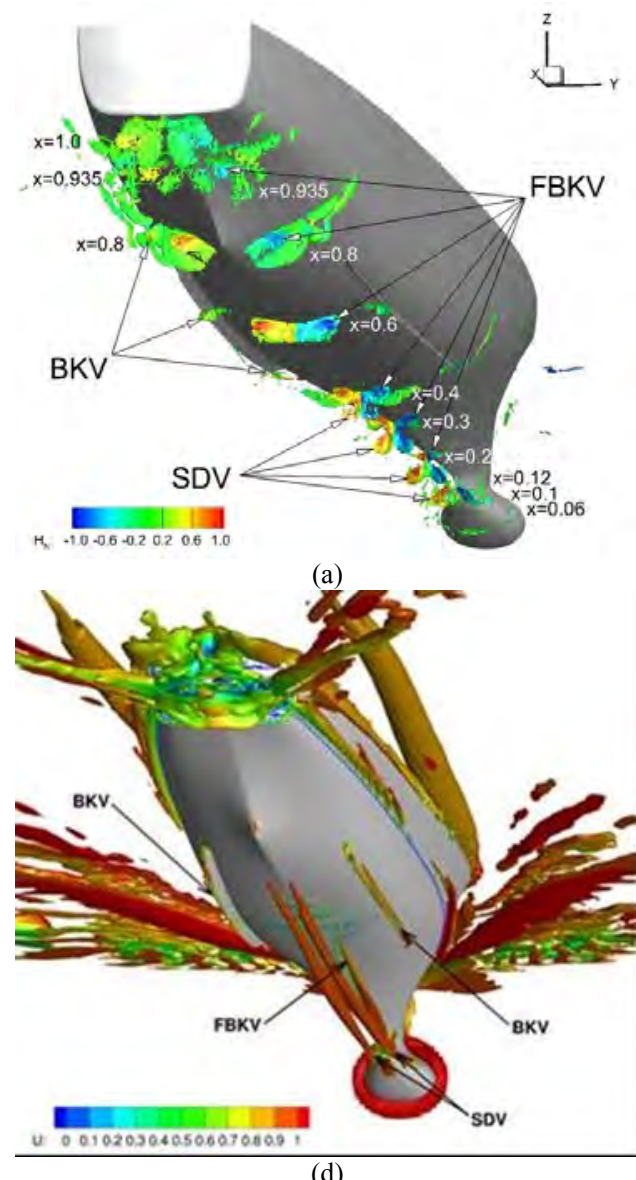

(d)

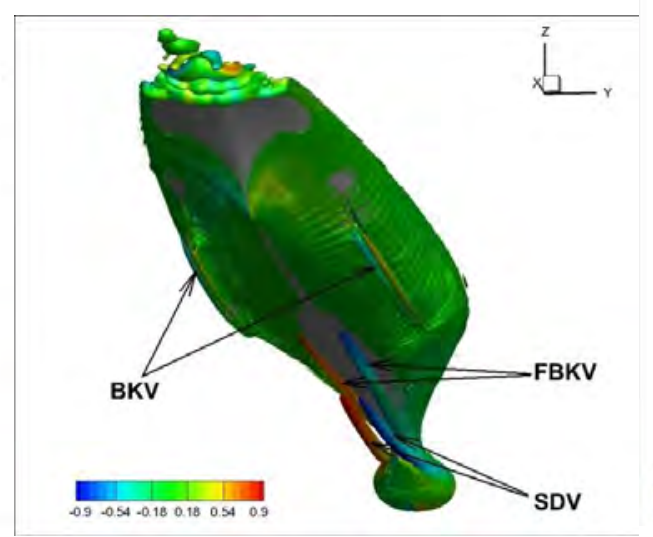

(b)

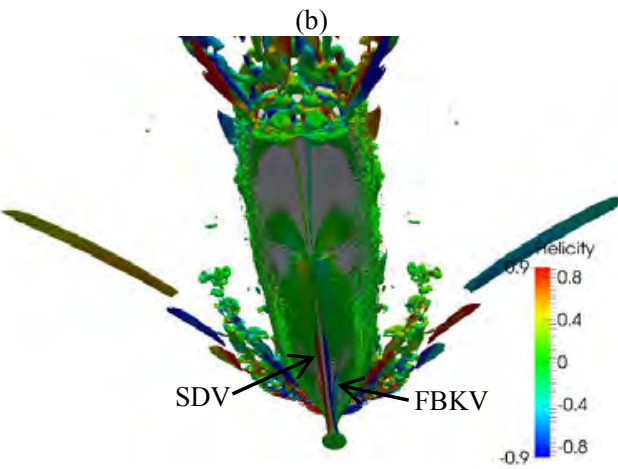

(e)

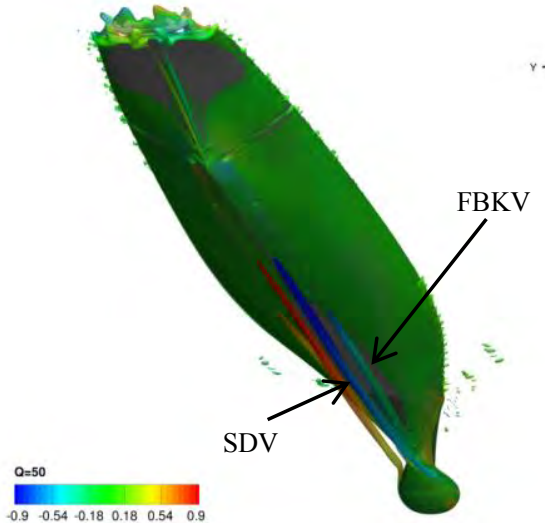

(c)

Figure 4: Iso-surfaces of $\mathrm{Q}=50$ colored by relative helicity obtained using (a) experiment in selected 3D volumes; (b) CFDShip-IOWA URANS; (c) ReFRESCO; (d) ISIS-CFD; (e) NavyFOAM; and (f) CFDShip-IOWA DES for straight-ahead $\beta=0$ condition.

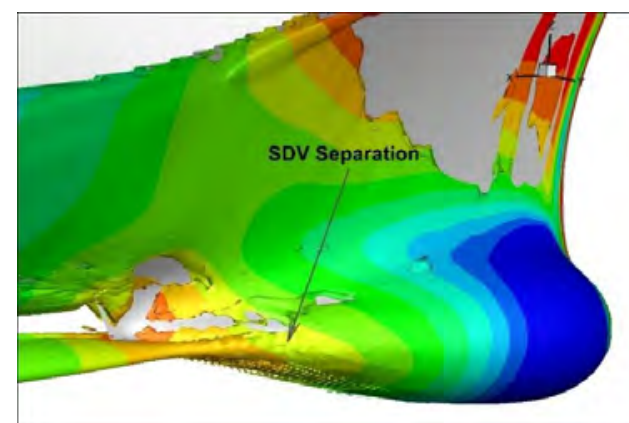

(a)

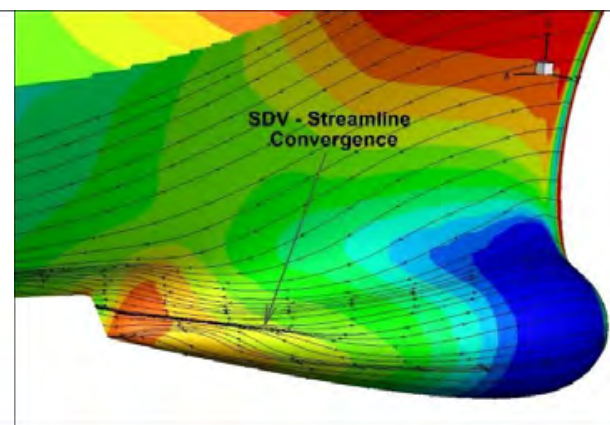

(b)

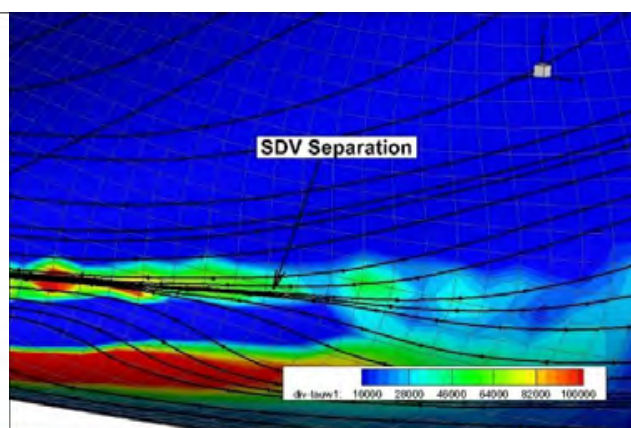

(c)

Figure 5: Zoomed in view of sonar dome show SDV flow separation predicted by CFDShip-Iowa URANS on $84 \mathrm{M}$ grid for straight-ahead $\beta=0$ condition. (a) Iso-surface of $\mathrm{Q}=100$ showing SDV separation, and (b) surface streamline showing onset of SDV. Both plots (a) and (b) are colored using pressure. (c) Contours of wall shear stress distribution of $\operatorname{div}\left(\tau_{\mathrm{w}}\right)$. 

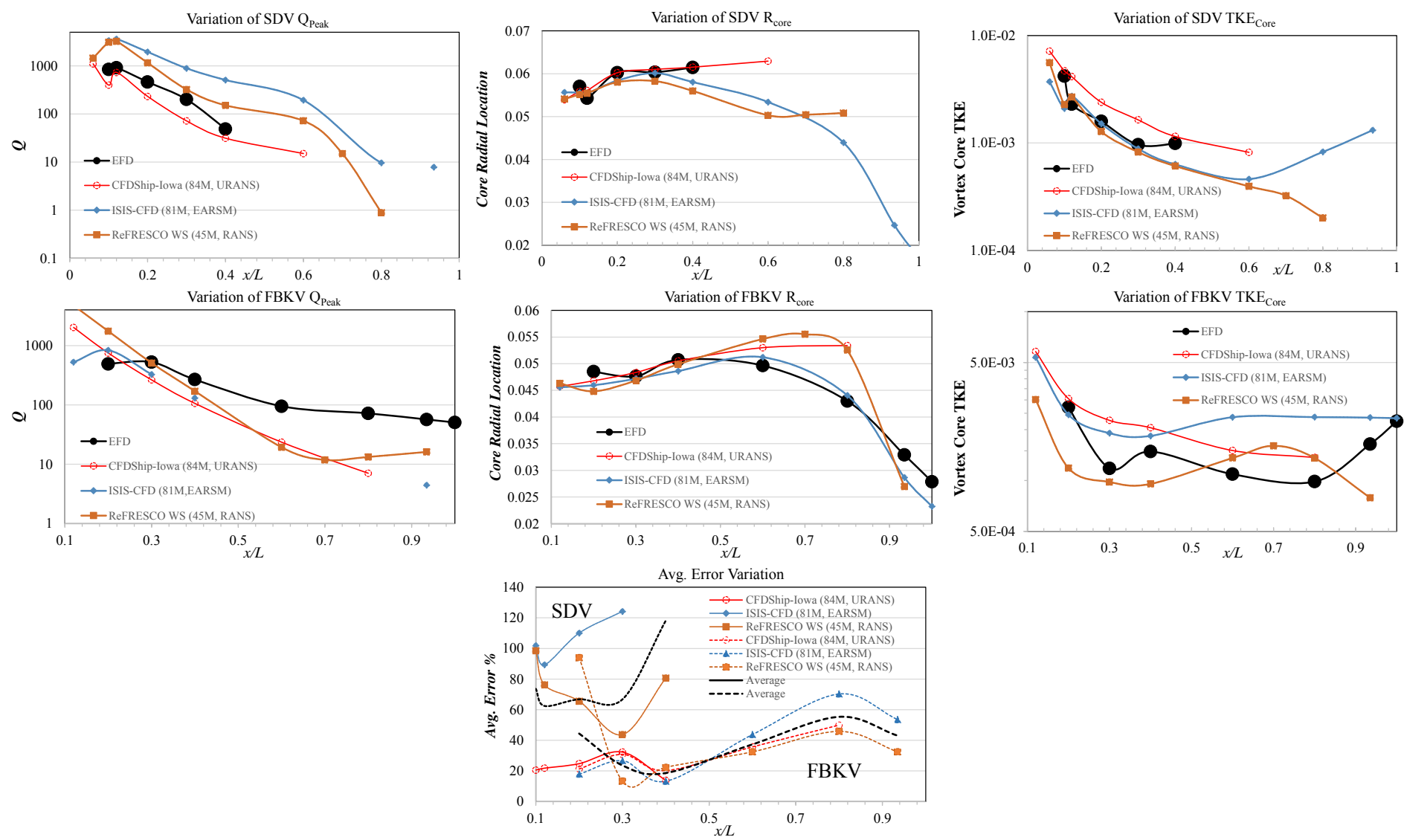

Figure 6: Axial variation of flow variables in SDV (Top) and FBKV (Middle) vortex core for straight-ahead $\beta=0$ condition predicted by CFDShipIowa; ReFRESCO and ISIS-CFD are compared with experiment. The bottom subfigure shows the axial variation of the average error prediction for both SDV and FBKV.

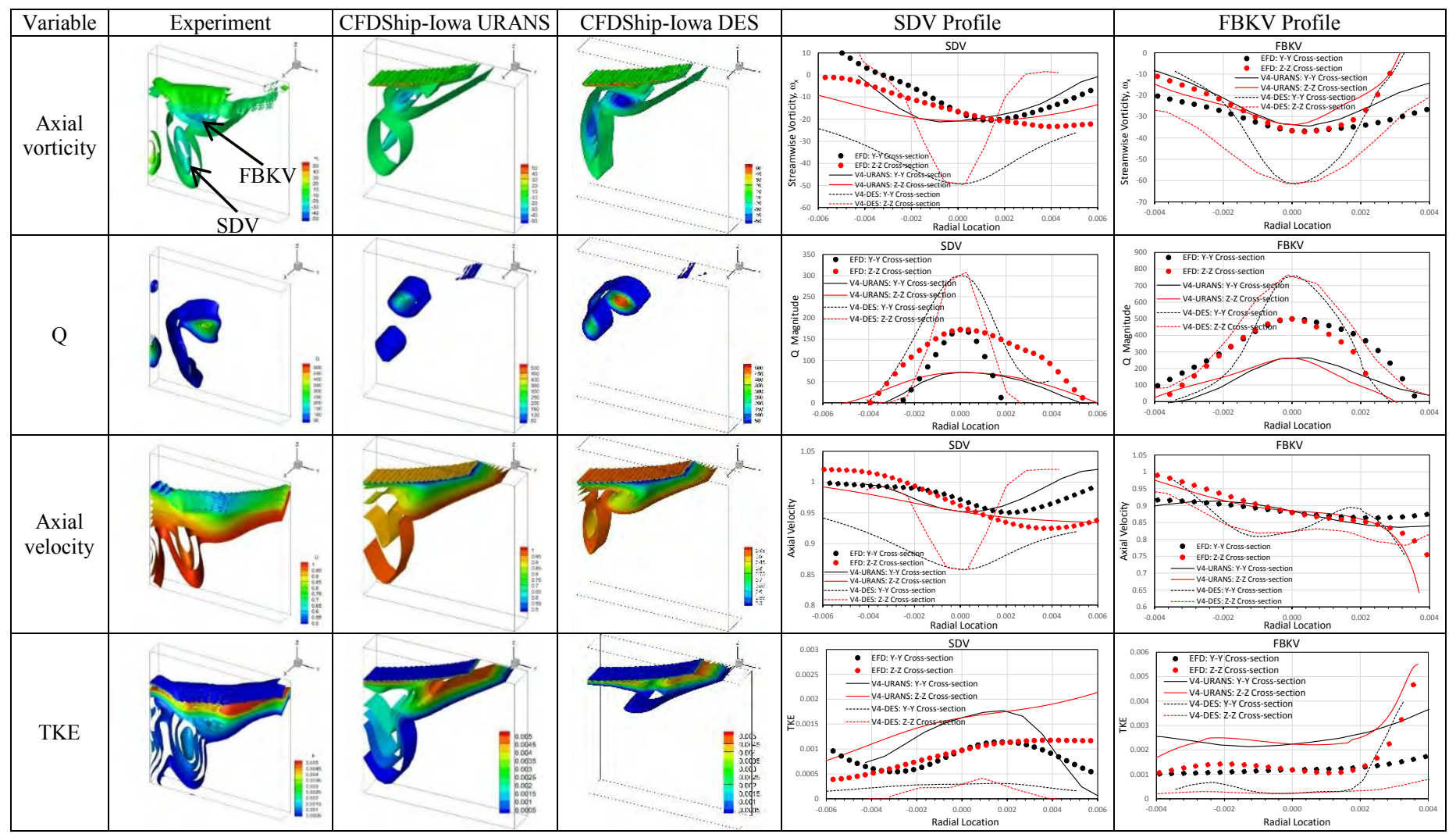

Figure 7: Volume plots and cross-plane profiles for axial velocity, Q, axial velocity and TKE for SDV and FBKV cores at $\mathrm{x} / \mathrm{L}=0.3$ for straightahead $\beta=0$ condition obtained using CFDShip-Iowa URANS and DES on 84M grid are compared with experiment. 


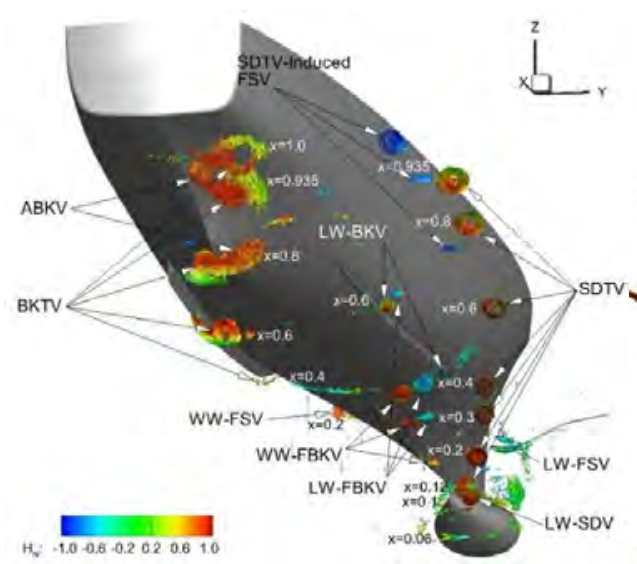

(a)

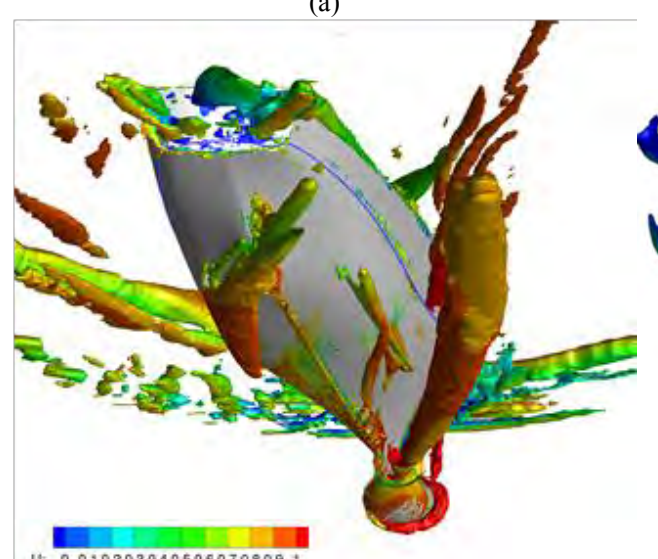

(d)

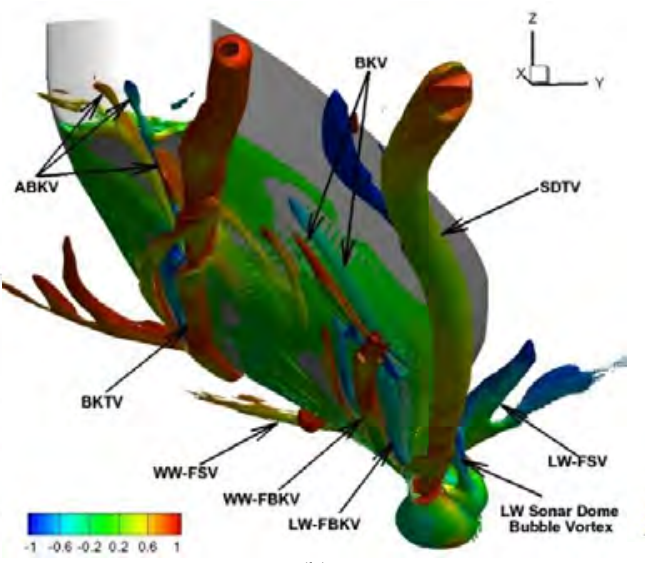

(b)

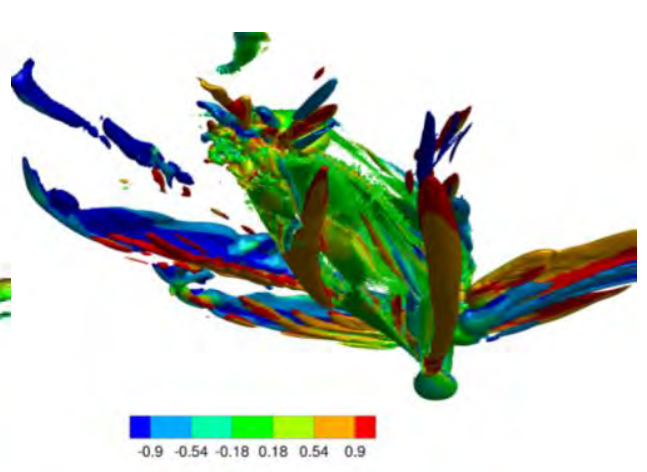

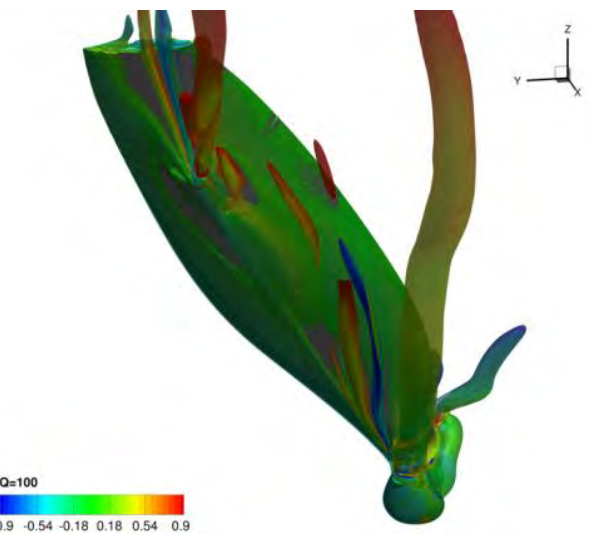

(c)

(e)

Figure 8: Iso-surfaces of $\mathrm{Q}=100$ colored by relative helicity obtained using: (a) experimental data at selected 3D volumes; (b) CFDShipIOWA; (c) ReFRESCO; (d) ISIS-CFD; and (e) NavyFOAM for static drift $\beta=20$ condition.

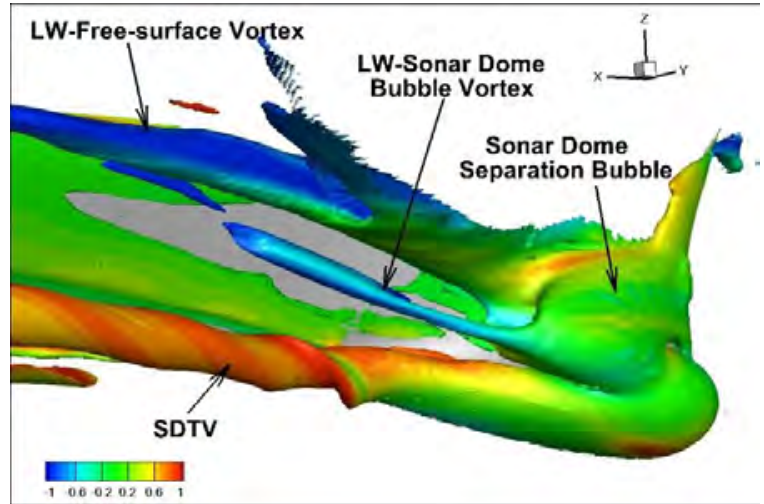

(a)

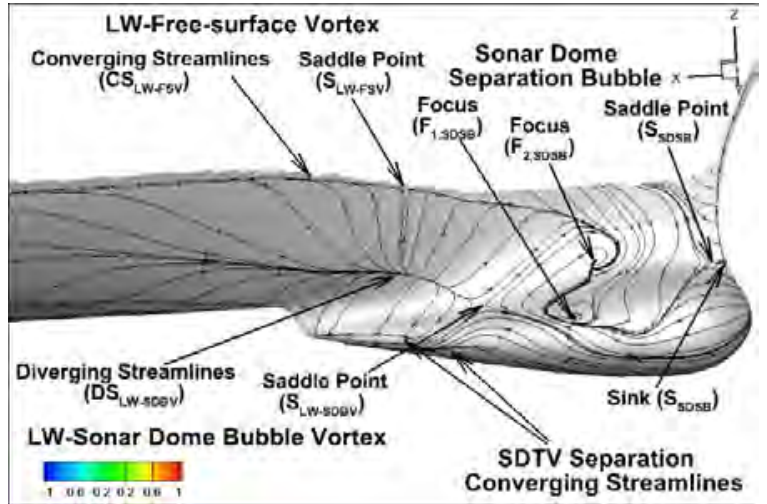

(b)

Figure 9: (a) Iso-surfaces of $Q=1000$ colored by relative helicity and (b) surface streamlines close to the leeward sonar dome surface obtained using averaged CFDShip-Iowa (DES 84M) solution for static drift $\beta=20$ condition. 

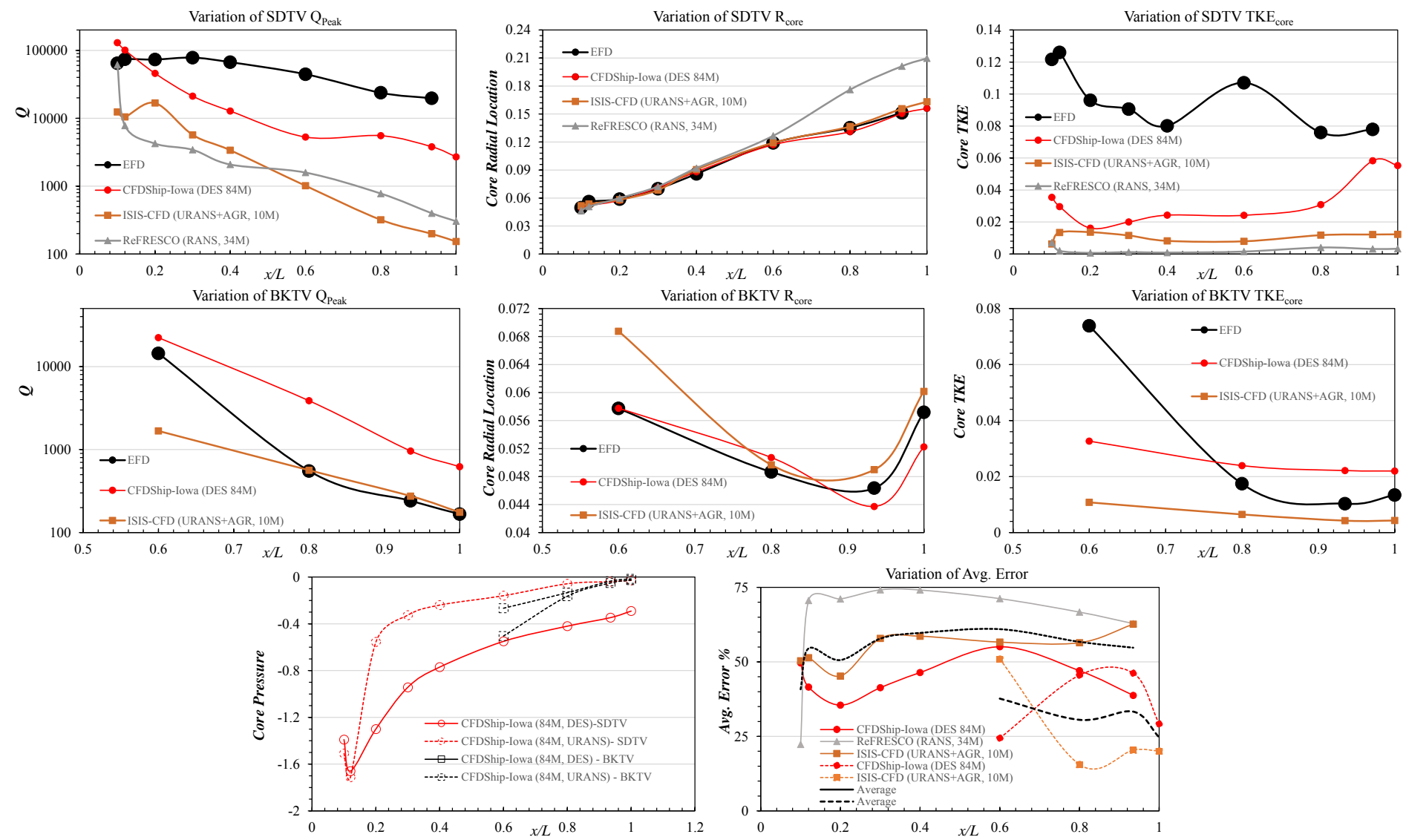

Figure 10: Axial variation of flow variables in SDTV (Top) and BKTV (Middle) vortex core predicted for static drift $\beta=20$ condition by CFDShipIowa, ISIS-CFD and ReFRESCO are compared with data. The bottom left subfigure shows the variation of core pressure predicted by CFDShip-Iowa for SDTV and BKTV. The bottom right subfigure shows the variation of the average error prediction vs axial location.

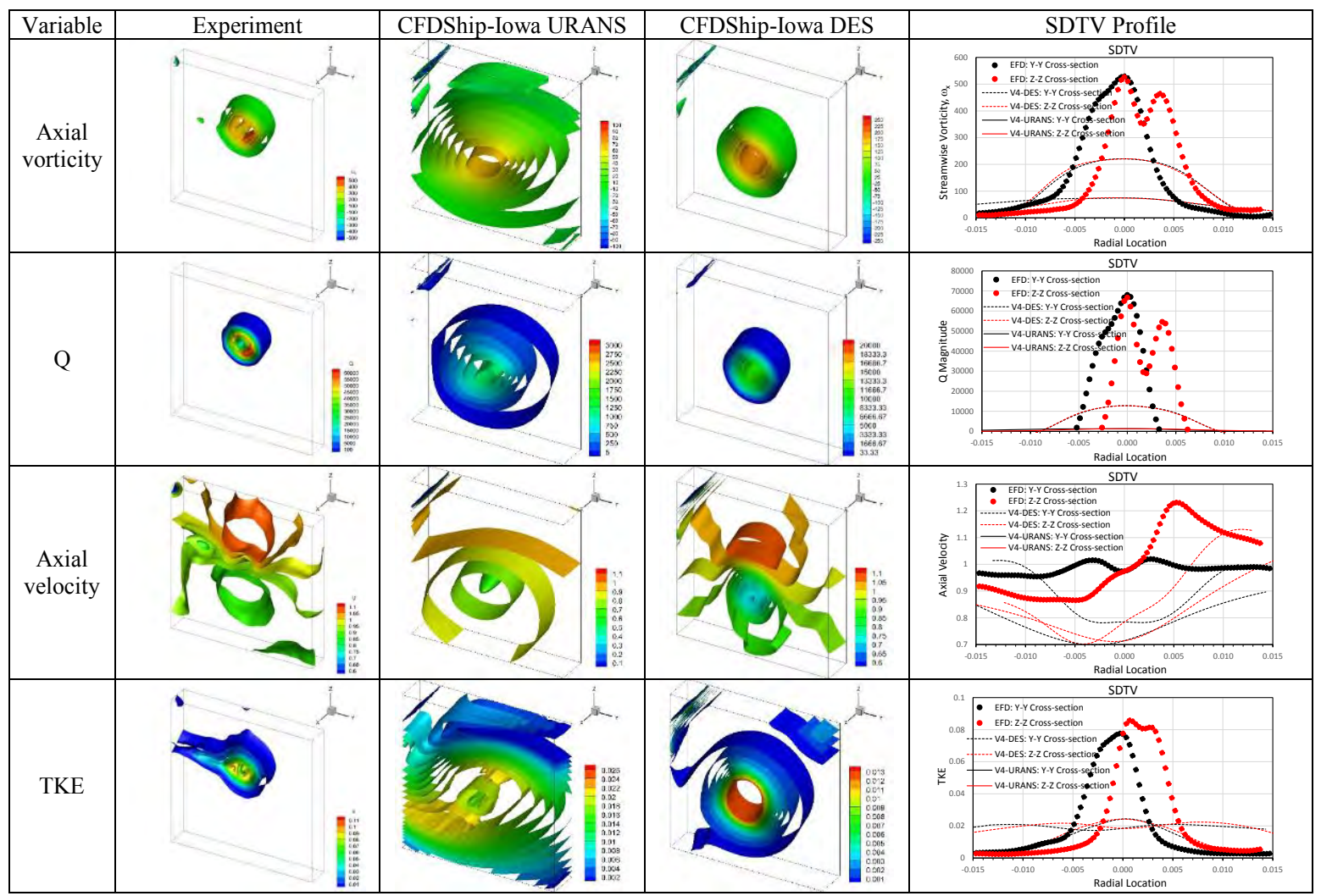

Figure 11: Volume plots and cross-plane profiles for axial velocity, $\mathrm{Q}$, axial velocity and TKE at $\mathrm{x} / \mathrm{L}=0.4$ in SDTV core for static drift $\beta=20$ condition obtained using CFDShip-Iowa URANS and DES on 84M grid are compared with experimental data. 


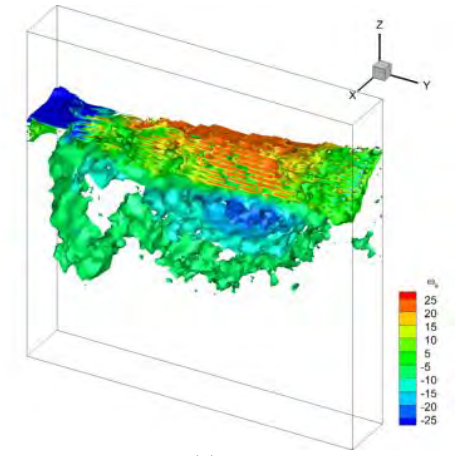

(a)

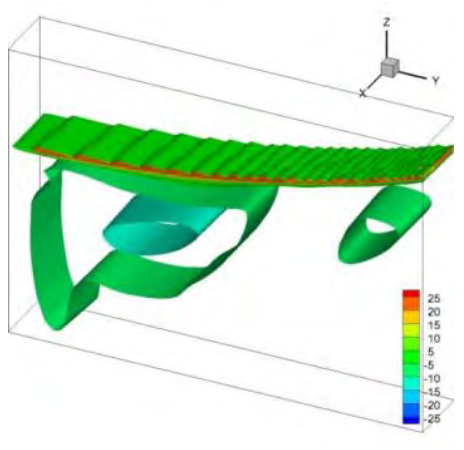

(b)

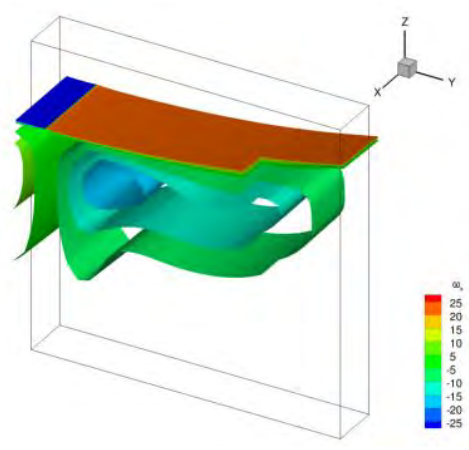

(c)

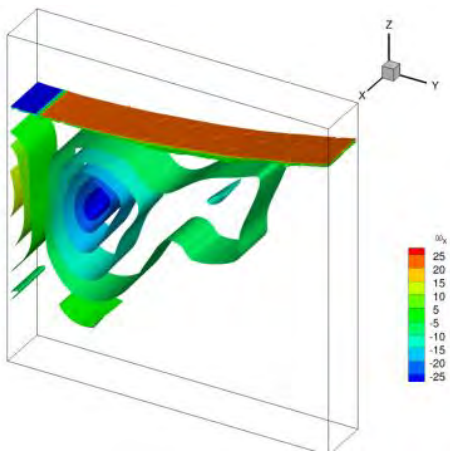

(d)

Figure 12: Iso-surface of axial vorticity structure for volume flow obtained using: (a) experiment; (b) CFDShip-IOWA; (c) ReFRESCO; and (d) ISIS-CFD at $x / L_{P P}=0.6$ for straight-ahead $\beta=0$ condition.

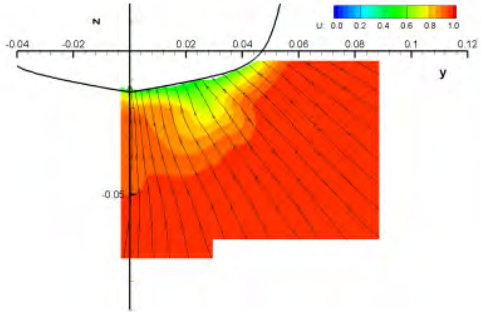

(a)

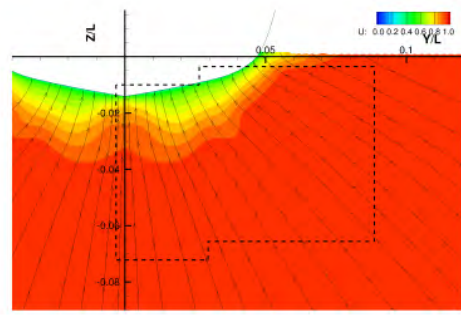

(d)

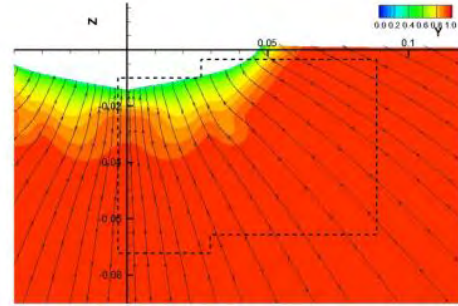

(b)

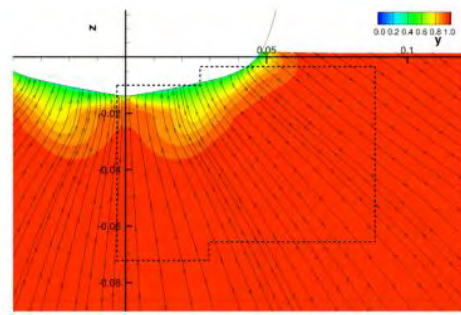

(e)

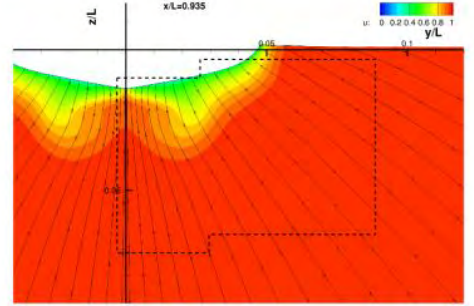

(c)

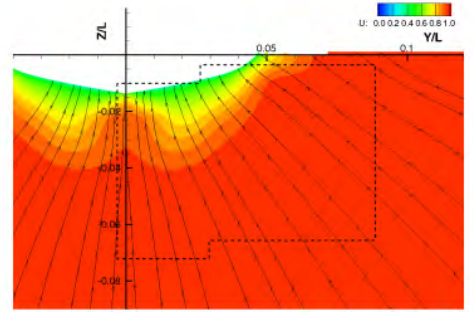

(f)

Figure 13: Contours of axial velocity and cross flow streamlines obtained using: (a) experiment; (b) CFDShip-IOWA; (c) ReFRESCO; (d) ISIS-CFD; (e) NavyFOAM and (f) STRARCCM+ at $x / L_{P P}=0.935$ for straight-ahead $\beta=0$ condition.

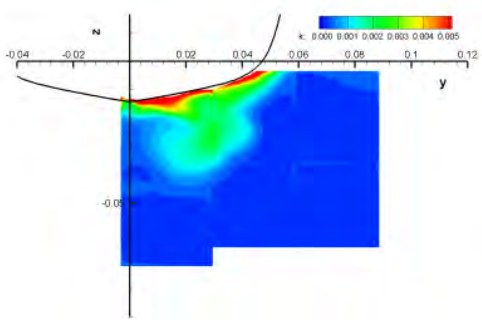

(a)

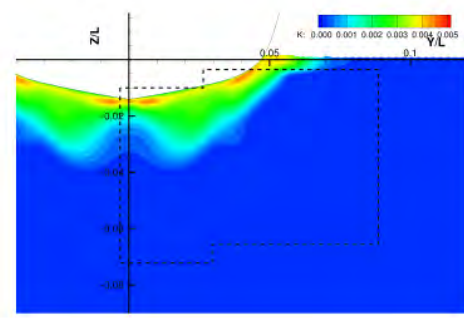

(d)

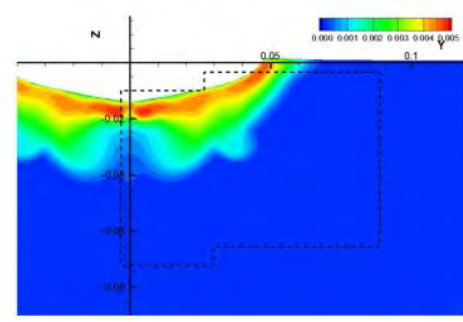

(b)

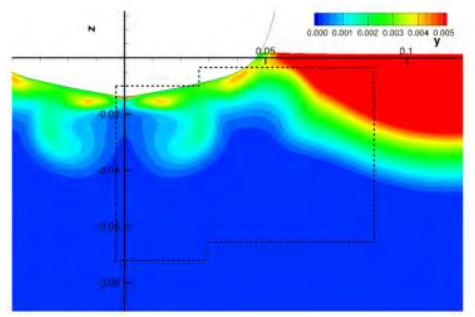

(e)

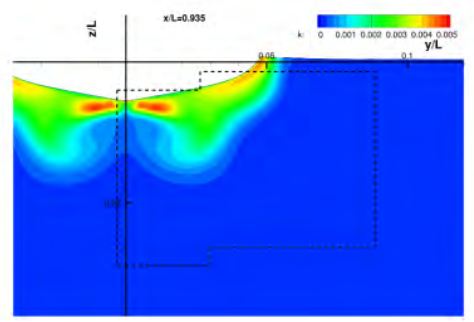

(c)

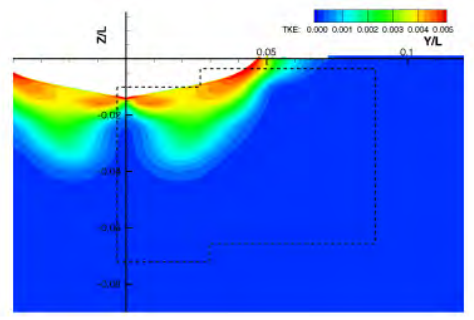

(f)

Figure 14: Contours of TKE obtained using: (a) experiment; (b) CFDShip-IOWA; (c) ReFRESCO; (d) ISIS-CFD; (e) NavyFOAM and (f) STRARCCM + at $x / L_{P P}=0.935$ for straight-ahead $\beta=0$ condition. 


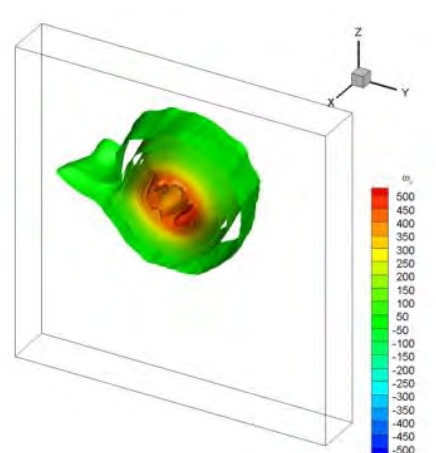

(a)

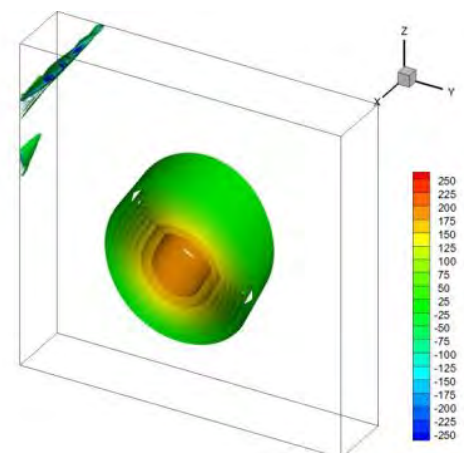

(b)

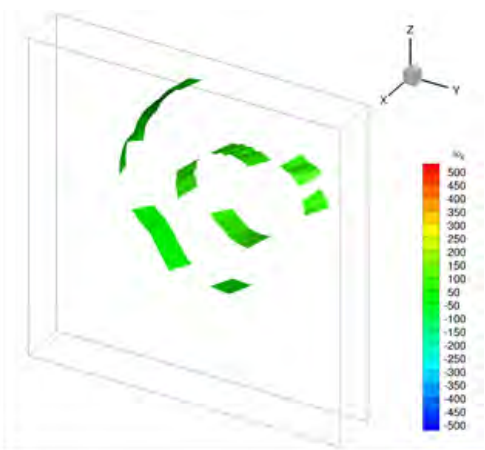

(c)

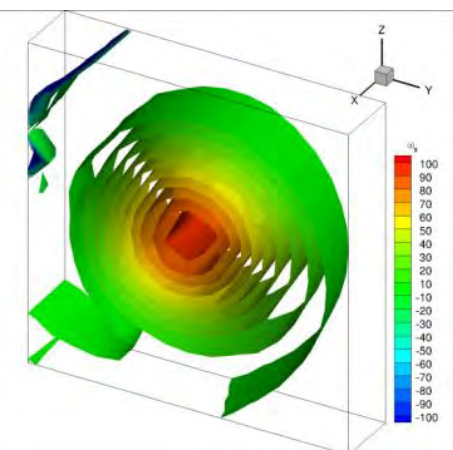

(d)

Figure 15: Iso-surface of vortical structure for volume flow around SDTV obtained using (a) experimental data; (b) CFDShip-IOWA; (c) ISIS-CFD; and (d) NavyFOAM at $x / L_{P P}=0.4$ for static drift $\beta=20$ condition.

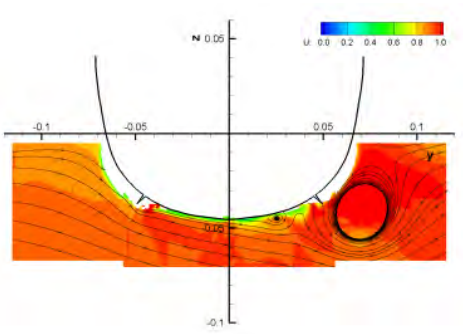

(a)

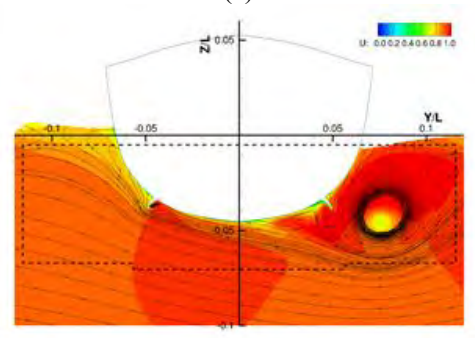

(e)

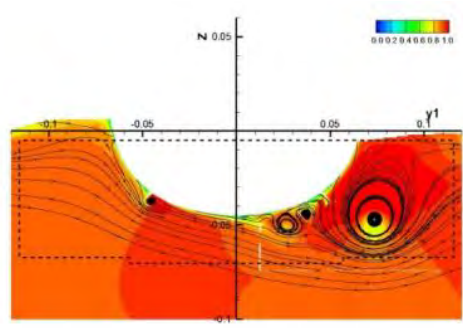

(b)

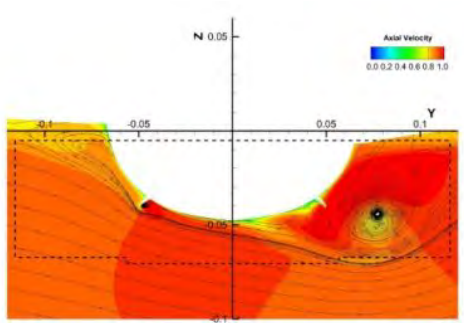

(c)

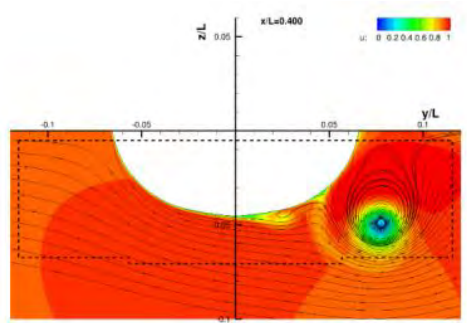

(d)

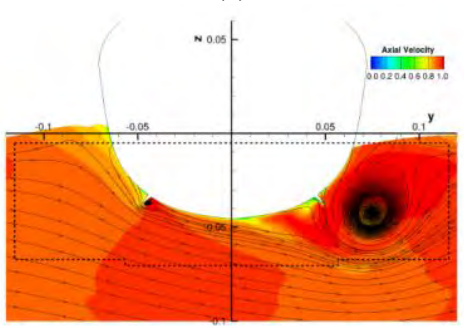

(f)

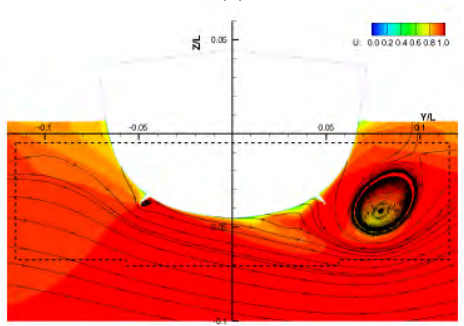

(g)

Figure 16: Contours of axial velocity and cross flow streamlines obtained using (a) experimental data; (b) CFDShip-IOWA (c) STAR-CCM+ (FORCE); (d) ReFRESCO; (e) ISIS-CFD; (f) NavyFOAM; and (f) STAR-CCM+ (NTUA) at $x / L_{P P}=0.4$ for static drift $\beta=20$ condition.

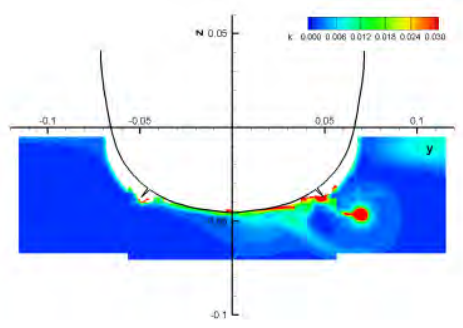

(a)

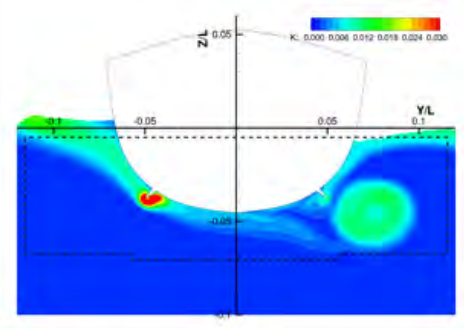

(e)

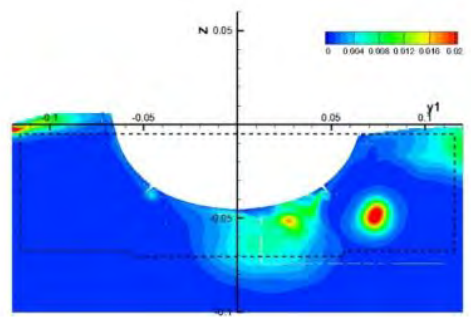

(b)

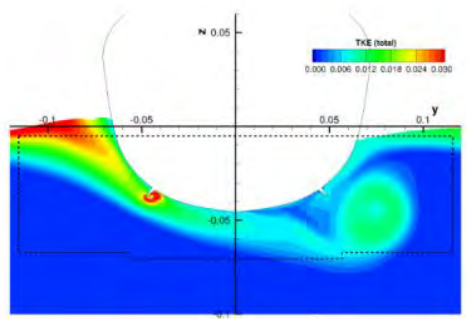

(f)

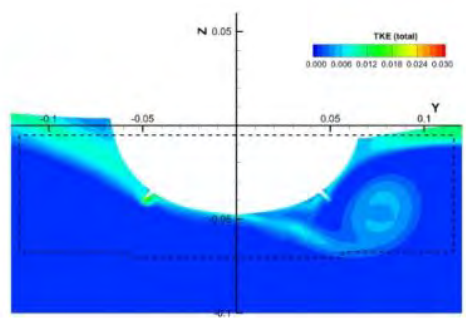

(c)

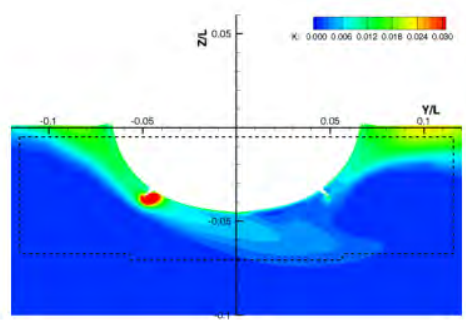

(g)

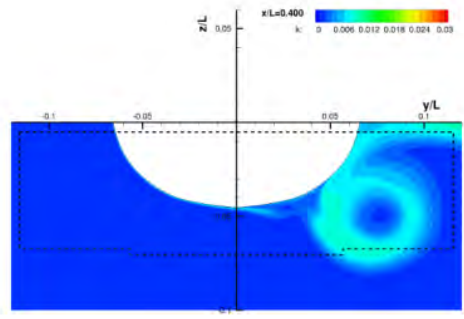

(d)

Figure 17: Contours of TKE obtained using (a) experimental data; (b) CFDShip-IOWA; (c) STAR-CCM+; (d) ReFRESCO RANS on 34M grid; (e) ISIS-CFD; (f) NavyFOAM; and (f) STAR-CCM+ (NTUA) at $x / L_{P P}=0.4$ for static drift $\beta=20$ condition. 


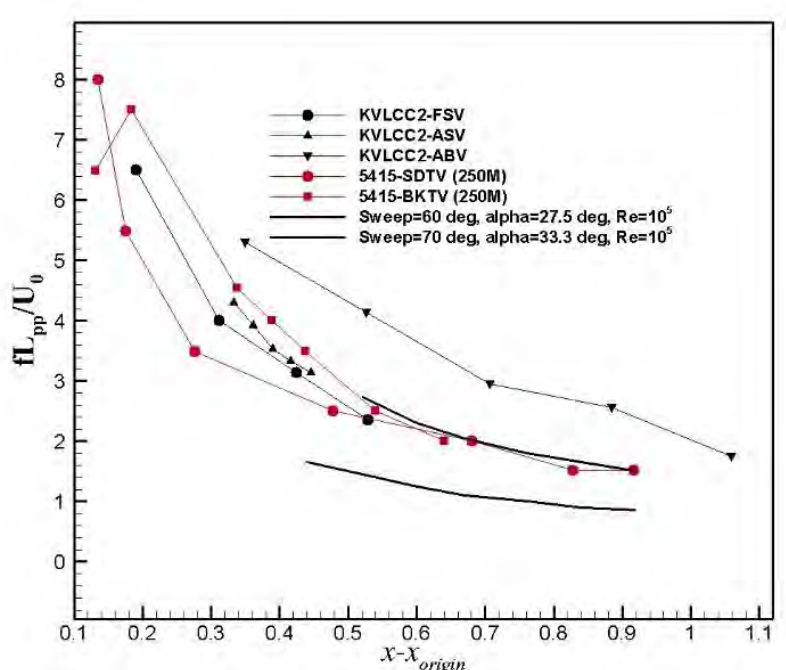

(a)

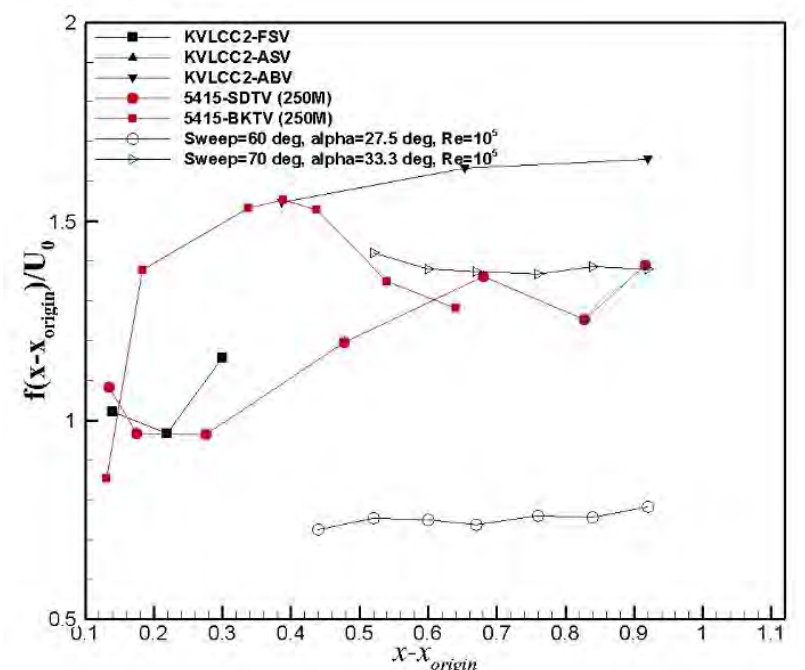

(b)

Figure 18: Variation of (a) dimensionless frequency and (b) product frequency and distance from origin as a function of streamwise distance along vortex core for SDTV and BKTV. The predictions are compared with KVLCC2 (Xing et al. 2012) and delta wing (Gursul, 1994) results.
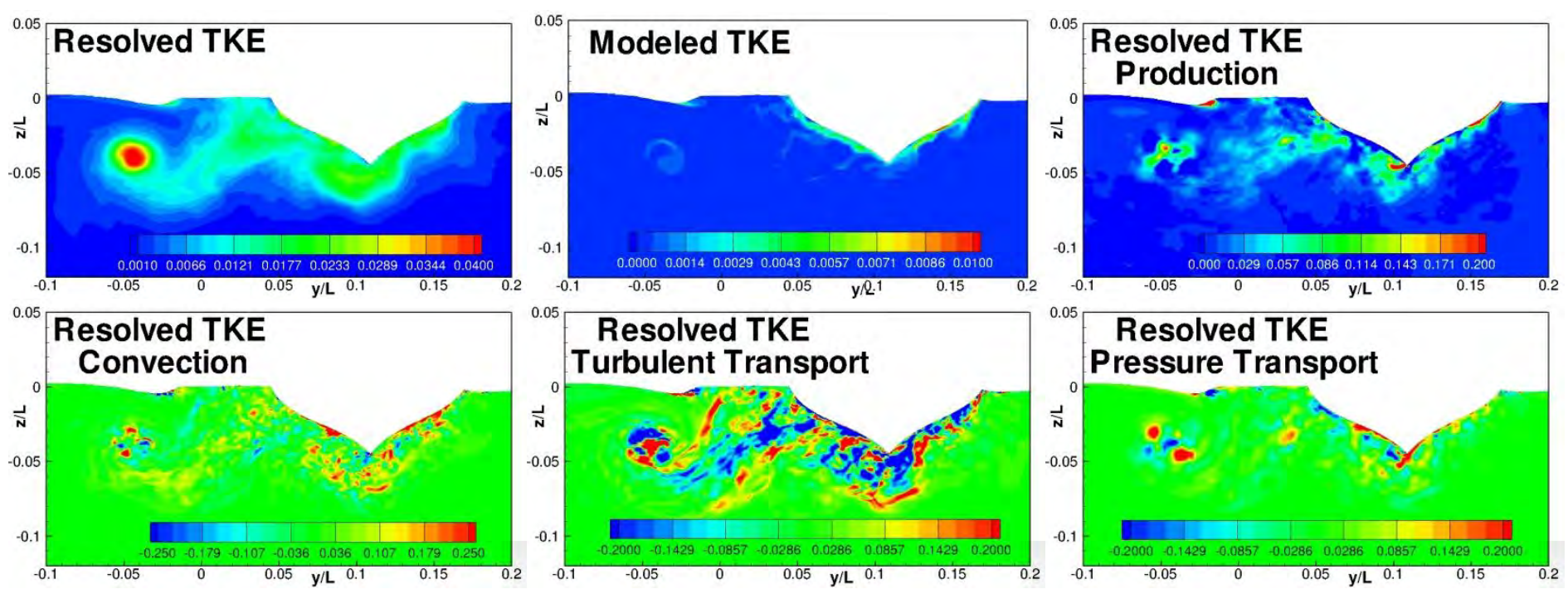

Figure 19: TKE budget at $\mathrm{x}_{\mathrm{e}} / \mathrm{L}=0.8$ obtained using CFDShip-Iowa DES on $250 \mathrm{M}$ grid for static drift $\beta=20$ condition. 